\title{
Forbidden oxygen lines in comets at various heliocentric distances ${ }^{\star}$
}

\author{
A. Decock, E. Jehin, D. Hutsemékers, and J. Manfroid
}

\begin{abstract}
Institut d'Astrophysique et Géophysique et Océanographie, Université de Liège, Allée du 6 août 17, 4000 Liège, Belgium e-mail: adecock@ulg.ac . be
\end{abstract}

Received 19 September 2012 / Accepted 12 April 2013

\section{ABSTRACT}

\begin{abstract}
We present a study of the three forbidden oxygen lines [OI] located in the optical region - i.e., $5577.339 \AA$ (the green line), $6300.304 \AA$ and $6363.776 \AA$ (the two red lines) - in order to better understand the production of these atoms in cometary atmospheres. The analysis is based on 48 high-resolution and high signal-to-noise spectra collected with UVES at the ESO VLT between 2003 and 2011 referring to 12 comets of different origins observed at various heliocentric distances. The flux ratio of the green line to the sum of the two red lines is evaluated to determine the parent species of the oxygen atoms by comparison with theoretical models. This analysis confirms that, at about $1 \mathrm{AU}, \mathrm{H}_{2} \mathrm{O}$ is the main parent molecule producing oxygen atoms. At heliocentric distances $>2.5 \mathrm{AU}$, this ratio changes rapidly, an indication that other molecules are starting to contribute. The most abundant species after $\mathrm{H}_{2} \mathrm{O}$ in the coma, $\mathrm{CO}_{\text {and }} \mathrm{CO}_{2}$, are good candidates, and the ratio is used to estimate their abundances. We found that the $\mathrm{CO}_{2}$ abundance relative to $\mathrm{H}_{2} \mathrm{O}$ in comet $\mathrm{C} / 2001 \mathrm{Q} 4$ (NEAT) observed at $4 \mathrm{AU}$ can be as high as $\sim 70 \%$. The intrinsic widths of the oxygen lines were also measured. The green line is on average about $1 \mathrm{~km} \mathrm{~s}^{-1}$ broader than the red lines, while the theory predicts that the red lines are broader. This might be due to the nature of the excitation source or to a contribution of $\mathrm{CO}_{2}$ as the parent molecule of the $5577.339 \AA$ Aline. At $4 \mathrm{AU}$, we found that the width of the green and red lines in comet $\mathrm{C} / 2001 \mathrm{Q} 4$ are the same, which could be explained if $\mathrm{CO}_{2}$ becomes the main contributor to the three [OI] lines at high heliocentric distances.
\end{abstract}

Key words. comets: general - techniques: spectroscopic - line: formation

\section{Introduction}

Comets are small bodies formed at the birth of the solar system 4.6 billion years ago. Since they did not evolve much, they are potential witnesses of the physical and chemical processes at play at the beginning of our solar system (Ehrenfreund \& Charnley 2000). Their status of "fossils" gives them a unique role to understand the origins of the solar system, not only from the physical and dynamical points of view but also from the chemical one (thanks to the knowledge of the compounds of the nucleus). When the comet gets closer to the Sun, the ices of the nucleus sublimate to form the coma (the comet atmosphere) where oxygen atoms are detected. Oxygen is an important element in the chemistry of the solar system given its abundance and its presence in many molecules including $\mathrm{H}_{2} \mathrm{O}$, which constitutes $80 \%$ of the cometary ices. Oxygen atoms are produced by the photodissociation of molecules coming from the sublimation of the cometary ices. Chemical reactions Eqs. (1) to (6) involve possible parent molecules (Bhardwaj \& Raghuram 2012; Festou \& Feldman 1981):

$$
\begin{aligned}
& \mathrm{H}_{2} \mathrm{O}+\mathrm{h} v \rightarrow \mathrm{H}_{2}+\mathrm{O}^{*}\left({ }^{1} \mathrm{D}\right) \\
& \mathrm{H}_{2} \mathrm{O}+\mathrm{h} v \rightarrow \mathrm{H}_{2}+\mathrm{O}^{*}\left({ }^{1} \mathrm{~S}\right) \\
& \mathrm{CO}_{2}+\mathrm{h} v \rightarrow \mathrm{CO}+\mathrm{O}^{*}\left({ }^{1} \mathrm{D}\right) \\
& \mathrm{CO}_{2}+\mathrm{h} v \rightarrow \mathrm{CO}+\mathrm{O}^{*}\left({ }^{1} \mathrm{~S}\right) \\
& \mathrm{CO}+\mathrm{h} v \rightarrow \mathrm{C}+\mathrm{O}^{*}\left({ }^{1} \mathrm{D}\right) \\
& \mathrm{CO}+\mathrm{h} v \rightarrow \mathrm{C}+\mathrm{O}^{*}\left({ }^{1} \mathrm{~S}\right) .
\end{aligned}
$$

* Based on observations made with ESO Telescope at the La Silla Paranal Observatory under programs ID 268.C-5570, 270.C-5043, 073.C-0525，274.C-5015，075.C-0355，080.C-0615，280.C-5053, 086.C-0958, and 087.C-0929.

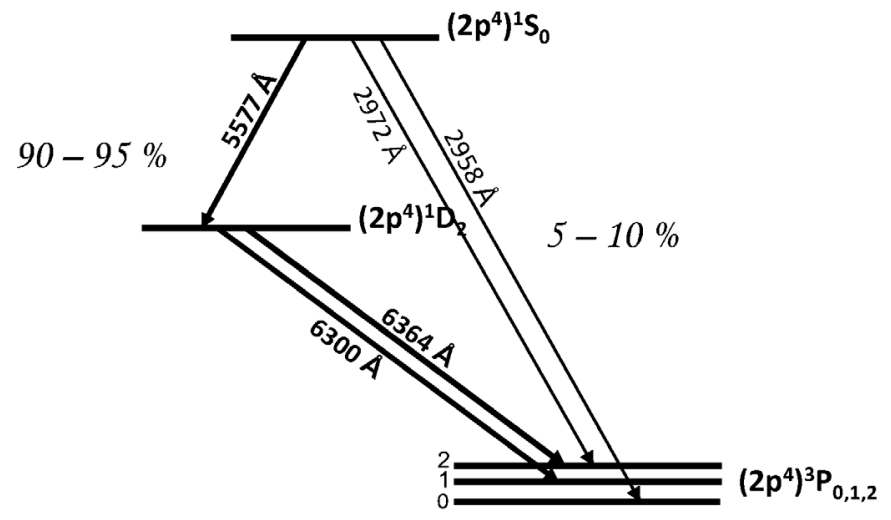

Fig. 1. Energy-level diagram for $[\mathrm{OI}]$ lines.

Oxygen atoms have been detected in comets through the three forbidden lines observed in emission at 5577.339 $\AA$ (the green line) and at $6300.304 \AA$ and $6363.776 \AA$ (the red lines) (Swings 1962). These lines come from the deexcitation of the upper state $\left(2 p^{4}\right)^{1} \mathrm{~S}_{0}$ to the $\left(2 \mathrm{p}^{4}\right)^{1} \mathrm{D}_{2}$ state for the $5577.339 \AA$ line and from the $\left(2 \mathrm{p}^{4}\right)^{1} \mathrm{D}_{2}$ state to the $\left(2 \mathrm{p}^{4}\right)^{3} \mathrm{P}_{1,2}$ states for the doublet red lines (see Fig. 1). The lifetime of the oxygen in the ${ }^{1} \mathrm{D}$ state is $\sim 110 \mathrm{~s}$ which is much longer than the lifetime of $\sim 1 \mathrm{~s}$ for the $\mathrm{O}\left({ }^{1} \mathrm{~S}\right)$. The measurement of the green line is more difficult owing to its fainter intensity and the many $\mathrm{C}_{2}$ lines located around it. One of the first theoretical studies was carried out by Festou \& Feldman (1981). They reviewed the production rate of $\mathrm{O}\left({ }^{1} \mathrm{~S}\right)$ and $\mathrm{O}\left({ }^{1} \mathrm{D}\right)$ obtained in the laboratory from $\mathrm{H}_{2} \mathrm{O}, \mathrm{CO}$, and $\mathrm{CO}_{2}$ photodissociations and measured the corresponding ${ }^{1} \mathrm{~S} /{ }^{1} \mathrm{D}$ ratio. 
Table 1. Emission rates and ${ }^{1} \mathrm{~S} /{ }^{1} \mathrm{D}$ ratios relevant to the quiet Sun obtained by Bhardwaj \& Raghuram (2012) for photodissociation reactions at $1 \mathrm{AU}$.

\begin{tabular}{lcccc}
\hline \hline \multirow{2}{*}{ Parents } & \multicolumn{2}{c}{ Emission rate $\left(\mathrm{s}^{-1}\right)$} & \multicolumn{2}{c}{ Ratio } \\
& $\mathrm{O}\left({ }^{1} \mathrm{~S}\right)$ & $\mathrm{O}\left({ }^{1} \mathrm{D}\right)$ & ${ }^{1} \mathrm{~S} /{ }^{1} \mathrm{D}$ & ${ }^{1} \mathrm{~S} /{ }^{1} \mathrm{D}^{a}$ \\
\hline $\mathrm{H}_{2} \mathrm{O}$ & $6.4 \times 10^{-8}$ & $8.0 \times 10^{-7}$ & 0.080 & $\sim 0.1$ \\
$\mathrm{CO}$ & $4.0 \times 10^{-8 b}$ & $5.1 \times 10^{-8}$ & 0.784 & $\sim 1$ \\
$\mathrm{CO}_{2}$ & $7.2 \times 10^{-7}$ & $1.2 \times 10^{-6}$ & 0.600 & $\sim 1$ \\
\hline
\end{tabular}

Notes. ${ }^{(a)}$ Ratios obtained by Festou \& Feldman (1981) are given in the last column for comparison. ${ }^{(b)}$ This rate comes from Huebner \& Carpenter (1979).

Recently, Bhardwaj \& Raghuram (2012) made a new model for oxygen atom emissions and calculated the production rate of $\mathrm{O}\left({ }^{1} \mathrm{~S}\right)$ and $\mathrm{O}\left({ }^{1} \mathrm{D}\right)$ for chemical reactions (1) to (6). The estimated ${ }^{1} \mathrm{~S} /{ }^{1} \mathrm{D}$ ratios are significantly different from Festou \& Feldman (1981) and are given in Table 1.

Up to now, little systematic researchwork has been done to study these lines at various heliocentric distances because the detection of the forbidden lines requires both high spectral and high spatial resolutions. Morrison et al. (1997) observed oxygen in comet C/1996 B2 (Hyakutake) with the 1-m Ritter Observatory telescope, Cochran \& Cochran (2001), and Cochran (2008) analyzed [OI] lines in the spectra of eight comets observed at the McDonald Observatory (see Bhardwaj \& Raghuram 2012 for a complete review of these measurements). The present paper reports the results obtained for a homogeneous set of high-quality spectra of 12 comets of various origins observed since 2003 with the UVES spectrograph mounted on the 8-m Kueyen telescope of the ESO VLT.

First, we measured the intensities of the three forbidden oxygen lines. Two ratios were evaluated: the ratio between the two red lines, $I_{6300} / I_{6364}$, and the ratio of the green line to the sum of the red lines, $I_{5577} /\left(I_{6300}+I_{6364}\right)$ which we denote as $\mathrm{G} / \mathrm{R}$ hereafter. The purpose of the latter is to determine the main parent molecule of the oxygen atoms by comparing our results with the Bhardwaj \& Raghuram (2012) effective excitation rates. We also followed how the $\mathrm{G} / \mathrm{R}$ ratio depends on the heliocentric distance by analyzing spectra of comets C/2001 Q4 (NEAT) and C/2009 P1 (Garradd) at small and large distances from the Sun.

Finally, we measured the full width at half maximum (FWHM) of the three lines. There is a longstanding debate on the FWHM measurement because the first observations made by Cochran (2008) contradict the theory. The intrinsic width of the green line is wider than the red ones, while the theory predicts the opposite (Festou 1981).

In this paper, we present the analysis of the three forbidden oxygen lines in various comets at different heliocentric distances. The observation of all the comets in our sample are explained in Sect. 2. In Sects. 3 and 4, details about data reduction and data analysis are described. Results of the two ratios and the FWHM of the [OI] lines are given in Sect. 5 followed by discussion in Sect. 6.

\section{Observations}

Our analysis is based on 12 comets listed in Table 2, considering the two components of $73 \mathrm{P} /$ Schwassmann-Wachmann 3 (B and C) as two comets. This sample is characterized by a wide diversity. These comets have various dynamical origins (external, new, Jupiter family, Halley type) and are observed at different heliocentric distances (from 0.68 AU to $3.73 \mathrm{AU}$ ). Some are split comets like 73P or did a close approach to Earth. Others have been observed at large heliocentric distances such as C/2001 Q4 and C/2009 P1. The observing material is made of a selection of 48 high signal-to-noise spectra obtained during the last ten years (Manfroid et al. 2009; Jehin et al. 2009, and references therein) with the cross-dispersed echelle spectrograph UVES (Dekker et al. 2000). A dichroic filter splits the light beam into two arms (a red one from 3000 to $5000 \AA$ and a blue one from 4200 to $11000 \AA$ ). In most observations, a slit of $0.45^{\prime \prime} \times 11^{\prime \prime}$ was used. With such a slit, the resolving power is $R=\lambda / \Delta \lambda=110000$ in the red arm where the three [OI] lines are observed. All spectra were recorded with nearly the same instrumental setting. Such high resolution is needed to isolate the [OI] emission lines from the telluric lines and other cometary lines $\left(\mathrm{C}_{2}, \mathrm{NH}_{2}\right)$ (see Fig. 2). The slit was always centered on the comet nucleus. For a comet located at $1 \mathrm{AU}$ from the Earth, the spatial area covered by the slit is approximatively $320 \mathrm{~km}$ by $8000 \mathrm{~km}$. The size of the slit for each individual spectrum is given in Table 3.

\section{Data reduction}

The 2D spectra were reduced with the UVES pipeline reduction program. For data obtained until 2008, the UVES pipeline (version 2.8.0) was used within the ESO-MIDAS environment to extract, for each order separately, the 2D spectra, bias-subtracted, flat-fielded, and wavelength-calibrated. ESO-MIDAS is a special software containing packages to reduce ESO data ${ }^{1}$. For a given setting, the orders were then merged using a weighting scheme to correct for the blaze function, computed from high S/N master flat-fields reduced in exactly the same way. This procedure leads to a good order merging. The post-2008 observations were reduced with the gasgano CPL ${ }^{2}$ interface (version 2.4.3) of the refurbished UVES pipeline that directly provides accurately merged $2 \mathrm{D}$ spectra. The $1 \mathrm{D}$ spectra were extracted by averaging the $2 \mathrm{D}$ ones, with cosmic rejection. Standard stars were similarly reduced and combined to derive instrumental response functions used to correct the 1D spectra. The wavelength calibration was computed using thorium argon (Th-Ar) spectra. The calibration revealed small position shifts in the lines because the Th-Ar spectra were usually taken in the day and not right after the comet observations. This shift was removed using nine cometary lines of $\mathrm{NH}_{2}$ in the vicinity of the red [OI] lines for which laboratory wavelengths are well known.

Before doing any measurement, the telluric absorption lines were removed and the solar continuum contribution subtracted using the BASS ${ }^{3}$ and Kurucz ${ }^{4}$ spectra. These two spectra are solar spectra, with atmospheric absorption lines for the BASS spectrum and without for the Kurucz one (2005). Around the red doublet line at $6300 \AA$, there are indeed telluric absorption lines mostly due to $\mathrm{O}_{2}$ molecules in the Earth's atmosphere, which could lead to an underestimate of the forbidden oxygen line intensity (see Fig. 3).

The comet solar continuum has three contributions. First and foremost is the one from the reflection of sunlight on the dust particles in the coma. Another one appears when the observations are made in or close to the twilight. A third contribution is,

\footnotetext{
http://www . eso.org/sci/software/esomidas/

2 http://www.eso.org/sci/software/gasgano/

3 http://bass2000.obspm.fr/solar_spect.php

4 http://kurucz .havard.edu/sun/irradiance2005/

irradthu.dat
} 
A. Decock et al.: [OI] lines in comets at various heliocentric distances

Table 2. Orbital characteristics of the 12 comets and their classification.

\begin{tabular}{|c|c|c|c|c|c|c|c|c|}
\hline Comet & $T_{\mathrm{p}}$ & $e$ & $a$ & $q$ & $i$ & $P$ & $T_{\mathrm{J}}$ & Type (L) \\
\hline C/2002 V1 (NEAT) & $18-02-2003$ & 0.99990 & 1010 & 0.10 & 82 & 32100 & 0.06 & EXT \\
\hline C/2002 X5 (Kudo-Fujikawa) & 29-01-2003 & 0.99984 & 1175 & 0.19 & 94 & 40300 & -0.03 & EXT \\
\hline C/2002 Y1 (Juels-Holvorcem) & $13-04-2003$ & 0.99715 & 250.6 & 0.71 & 104 & 3967 & -0.23 & EXT \\
\hline C/2001 Q4 (NEAT) & $16-05-2004$ & 1.00069 & - & 0.96 & 100 & - & - & NEW \\
\hline C/2002 T7 (LINEAR) & 23-04-2004 & 1.00048 & - & 0.61 & 161 & - & - & NEW \\
\hline C/2003 K4 (LINEAR) & $14-10-2004$ & 1.00030 & - & 1.02 & 134 & - & - & NEW \\
\hline 9P/Tempel 1 & 05-07-2005 & 0.51749 & 3.1 & 1.51 & 11 & 5.5 & 2.97 & $\mathrm{JF}$ \\
\hline 73P-C/Schwassmann-Wachmann 3 & 07-06-2006 & 0.69338 & 3.1 & 0.94 & 11 & 5.4 & 2.78 & JF \\
\hline 73P-B/Schwassmann-Wachmann 3 & 08-06-2006 & 0.69350 & 3.1 & 0.94 & 11 & 5.4 & 2.78 & JF \\
\hline $8 \mathrm{P} /$ Tuttle & 27-01-2008 & 0.81980 & 5.7 & 1.03 & 55 & 13.6 & 1.60 & $\mathrm{HF}$ \\
\hline 103P/Hartley 2 & $28-10-2010$ & 0.69500 & 3.5 & 1.06 & 14 & 6.5 & 2.64 & $\mathrm{JF}$ \\
\hline C/2009 P1 (Garradd) & 23-12-2011 & 1.00110 & - & 1.55 & 106 & - & - & NEW \\
\hline
\end{tabular}

Notes. $T_{\mathrm{p}}$ is the epoch of the perihelion (dd-mm-yyyy), $e$ the eccentricity, $a$ is related to the semi-major axis of the orbit (AU), $q$ is the perihelion distance (AU), $i$ represents the inclination on the ecliptic (degrees), $P$ is the period (years), $T_{\mathrm{J}}$ is the Tisserand parameter relative to Jupiter. The last data correspond to the classification of the comets according to Levison (1996): HF and JF mean "Halley family" and "Jupiter family", and they gather comets with short periods ( $<200$ years); EXT and NEW are respectively for external comets $(a<10000$ AU) and new comets $(a>10000 \mathrm{AU})$, which came from the Oort cloud.

in a few cases, the background radiation by the Moon. To remove these contaminations, we Doppler-shifted the BASS spectrum to the proper values and scaled the intensities until the solar features are completely removed. This provides the final spectrum, such as the one presented in Fig. 4 for comet 8P/Tuttle, free of any solar and telluric lines.

\section{Data analysis}

After the reduction and the correction of the data, we measured both the intensity and the FWHM of the three forbidden oxygen lines by fitting a Gaussian profile using the IRAF $^{5}$ software. The observed width is the convolution of the instrumental profile with the natural width:

$$
F W H M_{\text {observed }}(\lambda)=\sqrt{F W H M_{\text {intrinsic }}^{2}(\lambda)}+F W H M_{\text {instrumental }}^{2}(\lambda),(7)
$$

where the instrumental width corresponds to the width of the Th-Ar lines.

These measurements can only be performed with the best accuracy when the cometary oxygen lines are separated from the telluric [OI] lines well, i.e, when the geocentric velocitiy of the comet exceeds $15 \mathrm{~km} \mathrm{~s}^{-1}$. Using the deblend function of the splot package in IRAF, we could measure the [OI] lines for Doppler shifts as low as $7 \mathrm{~km} \mathrm{~s}^{-1}$.

\section{Results}

We measured the two ratios and the FWHM of the three [OI] lines. To reach such goals, a relative calibration using the well known UVES instrumental response curve provided by ESO was enough. Nevertheless, we investigate how to produce absolute flux-calibrated spectra in order to derive production rates of the oxygen parent species in a future work. Therefore, in this current study, the production rates of $\mathrm{H}_{2} \mathrm{O}$ to assess the activity of the comet have been taken from the literature.

\subsection{Intensity ratios}

The two measured intensity ratios, $I_{6300} / I_{6364}$ and $G / R=$ $I_{5577} /\left(I_{6300}+I_{6364}\right)$, are displayed for each spectrum in Figs. 5

\footnotetext{
5 IRAF is a tool for the reduction and the analysis of astronomical data (http://iraf.noao.edu).
}

and 6 respectively. When the collisional quenching is neglected, the intensity of a line is given by (Festou \& Feldman 1981)

$I=\tau_{\mathrm{p}}^{-1} \alpha \beta N$ photons $\mathrm{cm}^{-2} \mathrm{~s}^{-1}$

where $\tau_{\mathrm{p}}$ is the photodissociative lifetime of the parent, $\alpha$ the yield of photo-dissociation, $\beta$ corresponds to the branching ratio for the transition, and $N$ is the column density of the parent. Table 4 lists the line intensities $I$ (in ADU) and the ratios for all the spectra. Table 5 shows the standard deviation $(\sigma)$ of the ratio for all the spectra of a given comet. Since usually most of the spectra of the same comet are obtained during a short interval of time at similar heliocentric and geocentric distances, as well as the same exposure time, the standard deviation of these measurements provides a good estimate of the error. Indeed, this error includes the photon noise and errors coming from the telluric absorption and solar continuum corrections.

The red doublet ratio is remarkably similar for all comets. The average value over the whole sample of comets is $3.11 \pm 0.10$. The error corresponds to the standard deviation $(\sigma)$. The ratio agrees with the value of $3.09 \pm 0.12$ obtained by Cochran (2008) from a sample of eight comets but with better accuracy. Since both red lines are transitions from the same level to the ground state, $\tau_{\mathrm{p}}, \alpha$, and $N$ are equal in Eq. (8), and the intensity ratio $I_{6300} / I_{6364}$ is equivalent to the branching ratio $\beta_{6300} / \beta_{6364}$. Our results are indeed in very good agreement with the theoretical value of the branching ratio of 3.096 computed for the quantum mechanics (Galavis et al. 1997). Storey \& Zeippen (2000) have published a new theoretical value of 2.997 taking relativistic effects into account, which should be an improvement over previous determinations. Higher value derived from cometary spectra could point to a systematic error due to a small blend of the $6300.304 \AA$ [OI] line with an unidentified cometary feature. To check that these systematics are present, we first took the average red line ratio of the three comets at large heliocentric distances (C/2001 Q4, C/2003 K4, and C/2009 P1) since most of the fluorescence lines and then their contamination disappear far from the Sun. We obtained an average ratio of $3.13 \pm 0.07$. Second, we computed the same ratio, but this time for the terrestrial nightglow, and found a ratio equal to $3.20 \pm 0.05$ obtained again at the greatest cometary distances. These measurements agree better with the Galavis et al. (1997) value, but the error are too large to discard Storey \& Zeippen (2000) so we cannot exclude a systematic error. 

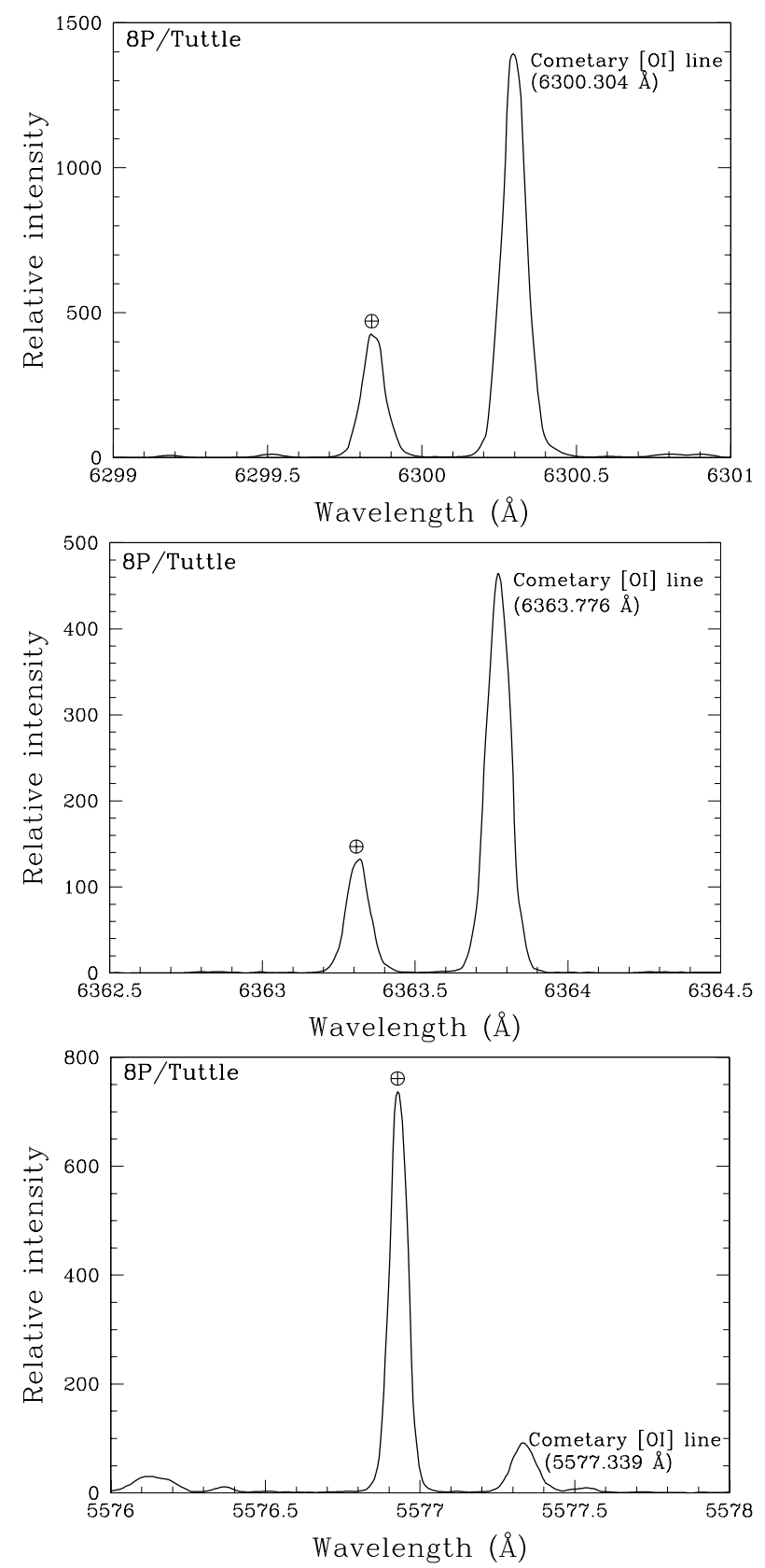

Fig. 2. The $6300.304 \AA, 6363.776 \AA$ and $5577.339 \AA$ oxygen lines (telluric $(\oplus)$ and cometary) in a $3600 \mathrm{~s}$ spectrum of comet $8 \mathrm{P} /$ Tuttle obtained in March 2008. Thanks to the high resolution of the UVES spectrograph, the cometary and telluric lines are well separated when the Doppler shift is larger than $15 \mathrm{~km} \mathrm{~s}^{-1}$. These spectra are representative of the quality of the sample.

The $\mathrm{G} / \mathrm{R}$ ratio has an average value of $0.11 \pm 0.07$ for the complete sample (see Fig. 6). This result agrees with the value of $0.09 \pm 0.01$ found by Cochran (2008). The dispersion is higher because it includes comets at large heliocentric distances that have different $\mathrm{G} / \mathrm{R}$ ratios. If we only take comets at $<2.5 \mathrm{AU}$ into account, the average and dispersion are equal to $0.09 \pm 0.02$. This leads to the conclusion that $\mathrm{H}_{2} \mathrm{O}$ is the main parent molecule producing oxygen atoms according to Bhardwaj \& Raghuram (2012) values (Table 1).

To see whether the quenching could play a role in our results, we computed the $\mathrm{G} / \mathrm{R}$ ratio with respect to the water production rates (Fig. 7). The water production rates of each comet has been compiled from the literature (cf. Table 8). Since those comets have been observed under different conditions (of production rates and heliocentric distances), the lack of trend in this analysis suggests that quenching is negligible and does not significantly affect our main conclusions. However, we have started to work on a model based on the Bhardwaj \& Raghuram (2012) paper and on Monte Carlo simulations to estimate the quenching. A careful computation of the quenching is not an easy task, and we will give our new results, including a detailed discussion of the quenching, in a forthcoming paper.

\subsection{Line widths}

Table 6 lists the intrinsic line velocity widths $\left(\operatorname{FWHM}(\mathrm{v})\left(\mathrm{km} \mathrm{s}^{-1}\right)\right)$. The $\operatorname{FWHM}(v)$ is obtained from the $\mathrm{FWHM}_{\text {intrinsic }}(\lambda)$ given in the Eq. (7) using the relation

$F W H M(v)=\frac{F W H M_{\text {intrinsic }}(\lambda) c}{\lambda_{[\mathrm{OI}]} 2 \sqrt{\ln 2}}$,

where $\lambda_{[\mathrm{OI}]}$ corresponds to the wavelength of the considered oxygen line, and $c$ is the speed of light $\left(\mathrm{km} \mathrm{s}^{-1}\right)$. The error on the [OI] line widths of each comet is given by the standard deviation of the $\mathrm{N}$ spectra (cf. Table 7). This error is about $5 \%$ for the red lines and $10 \%$ for the green line, which is fainter. The two red line widths are equal within the errors $\left(1.61 \pm 0.34 \mathrm{~km} \mathrm{~s}^{-1}\right.$ for the $6300 \AA$ line and $1.56 \pm 0.54 \mathrm{~km} \mathrm{~s}^{-1}$ for the $6364 \AA$ line), which is not a surprise since both lines are transitions from the $\mathrm{O}\left({ }^{1} \mathrm{D}\right)$ state to the ground state (Fig. 8). This result is also consistent with the values of Cochran (2008) $\left(1.22 \pm 0.36 \mathrm{~km} \mathrm{~s}^{-1}\right)$. Our mean value of the [OI] cometary green line is wider than the red lines and equal to $2.44 \pm 0.28 \mathrm{~km} \mathrm{~s}^{-1}$. This peculiarity has already been noticed by Cochran (2008), who found a mean velocity of $2.49 \pm 0.36 \mathrm{~km} \mathrm{~s}^{-1}$, in good agreement with our value. Figures 9-11 present the width of the three [OI] cometary lines, the three [OI] telluric lines, and the width of some representative neighboring cometary lines for comparison: $\mathrm{NH}_{2}$ lines in the red region and $\mathrm{C}_{2}$ lines in the green region. The intrinsic average widths of the cometary $\mathrm{NH}_{2}$ and $\mathrm{C}_{2}$ lines are, respectively, $1.00 \pm 0.20 \mathrm{~km} \mathrm{~s}^{-1}$ and $1.28 \pm 0.17 \mathrm{~km} \mathrm{~s}^{-1}$ and correspond well to what is expected for the gas expansion velocity in the coma at $1 \mathrm{AU}$. The ejection velocity due to the extra energy coming from the photodissociation of the parent molecule should be represented as the excess velocity that results from the subtraction of the $\mathrm{NH}_{2}$ or $\mathrm{C}_{2}$ cometary lines to the [OI] lines. Considering this, we find an average ejection velocities equal to $0.48 \pm 0.16 \mathrm{~km} \mathrm{~s}^{-1}$ for the red lines and $1.17 \pm 0.29 \mathrm{~km} \mathrm{~s}^{-1}$ for the green line. This analysis and Fig. 12 show clearly that the [OI] cometary green line is wider than the red lines. This width could be explained if the excess energy for the formation of the $\mathrm{O}\left({ }^{1} \mathrm{~S}\right)$ state is greater than for the $\mathrm{O}\left({ }^{1} \mathrm{D}\right)$ state. Contrary to what is observed, theoretical models using Ly- $\alpha$ photons as excitation source give an excess velocity of $1.6 \mathrm{~km} \mathrm{~s}^{-1}$ for the $\mathrm{O}\left({ }^{1} \mathrm{~S}\right)$ state and a value of $1.8 \mathrm{~km} \mathrm{~s}^{-1}$ for $\mathrm{O}\left({ }^{1} \mathrm{D}\right)$ in the case of water photodissociation (Festou 1981).

In Figs. 13 and 14, we compare the velocity of the three oxygen lines and the $\mathrm{H}_{2} \mathrm{O}$ production rate from the literature (cf. Table 8). We find that the velocity of the green oxygen line slightly increases with the water production rate. In Figs. 15 and 16, we provide similar plots for the velocities of $\mathrm{C}_{2}$ and $\mathrm{NH}_{2}$. The results agree with Tseng et al. (2007) but the error bars are large. Indeed, since the resolution of the spectrometer is at the limit for measuring the line broadening, we cannot improve their relation. 
Table 3. Individual spectra.

\begin{tabular}{|c|c|c|c|c|c|c|c|c|}
\hline Comet & $\mathrm{JD}-2450000.5$ & $r(\mathrm{AU})$ & $\dot{r}\left(\mathrm{~km} \mathrm{~s}^{-1}\right)$ & $\Delta(\mathrm{AU})$ & $\dot{\Delta}\left(\mathrm{km} \mathrm{s}^{-1}\right)$ & Exptime (s) & Slit $\left(" x^{\prime \prime}\right)$ & Slit $(\mathrm{km} \times \mathrm{km})$ \\
\hline C/2002 V1 (NEAT) & 2647.037 & 1.22 & -36.51 & 0.83 & 7.88 & 2100 & $0.45 \times 11.00$ & $271 \times 6622$ \\
\hline C/2002 V1 (NEAT) & 2647.062 & 1.22 & -36.53 & 0.83 & 7.99 & 2100 & $0.45 \times 11.00$ & $271 \times 6622$ \\
\hline C/2002 V1 (NEAT) & 2649.031 & 1.18 & -37.11 & 0.84 & 8.29 & 2100 & $0.45 \times 11.00$ & $274 \times 6702$ \\
\hline C/2002 V1 (NEAT) & 2649.056 & 1.18 & -37.11 & 0.84 & 8.33 & 1987 & $0.45 \times 11.00$ & $274 \times 6702$ \\
\hline C/2002 V1 (NEAT) & 2719.985 & 1.01 & 39.76 & 1.63 & 42.02 & 600 & $0.45 \times 11.00$ & $532 \times 13004$ \\
\hline C/2002 X5 (Kudo-Fujikawa) & 2705.017 & 1.06 & 37.01 & 0.99 & 29.34 & 1800 & $0.45 \times 11.00$ & $323 \times 7898$ \\
\hline C/2002 X5 (Kudo-Fujikawa) & 2705.039 & 1.07 & 37.00 & 0.99 & 29.40 & 1800 & $0.45 \times 11.00$ & $323 \times 7898$ \\
\hline C/2002 X5 (Kudo-Fujikawa) & 2705.060 & 1.07 & 36.99 & 0.99 & 29.45 & 1800 & $0.45 \times 11.00$ & $323 \times 7898$ \\
\hline C/2002 Y1 (Juels-Holvorcem) & 2788.395 & 1.14 & 24.09 & 1.56 & -7.24 & 1800 & $0.40 \times 11.00$ & $453 \times 12446$ \\
\hline C/2002 Y1 (Juels-Holvorcem) & 2788.416 & 1.14 & 24.09 & 1.56 & -7.21 & 1800 & $0.40 \times 11.00$ & $453 \times 12446$ \\
\hline C/2002 Y1 (Juels-Holvorcem) & 2789.394 & 1.16 & 24.18 & 1.55 & -7.21 & 1800 & $0.40 \times 11.00$ & $450 \times 12366$ \\
\hline C/2002 Y1 (Juels-Holvorcem) & 2789.415 & 1.16 & 24.19 & 1.55 & -7.18 & 1800 & $0.40 \times 11.00$ & $450 \times 12366$ \\
\hline C/2001 Q4 (NEAT) & 2883.293 & 3.73 & -18.80 & 3.45 & -25.42 & 4500 & $0.45 \times 11.00$ & $1126 \times 27524$ \\
\hline C/2001 Q4 (NEAT) & 2883.349 & 3.73 & -18.80 & 3.45 & -25.32 & 4500 & $0.45 \times 11.00$ & $1126 \times 27524$ \\
\hline C/2001 Q4 (NEAT) & 2889.236 & 3.67 & -18.91 & 3.36 & -23.67 & 7200 & $0.45 \times 11.00$ & $1097 \times 26806$ \\
\hline C/2001 Q4 (NEAT) & 2889.320 & 3.66 & -18.91 & 3.36 & -23.54 & 7200 & $0.45 \times 11.00$ & $1097 \times 26806$ \\
\hline C/2002 T7 (LINEAR) & 3131.421 & 0.68 & 15.83 & 0.61 & -65.62 & 1080 & $0.44 \times 12.00$ & $195 \times 5309$ \\
\hline C/2002 T7 (LINEAR) & 3151.976 & 0.94 & 25.58 & 0.41 & 54.98 & 2678 & $0.30 \times 12.00$ & $89 \times 3568$ \\
\hline C/2002 T7 (LINEAR) & 3152.036 & 0.94 & 25.59 & 0.42 & 55.20 & 1800 & $0.30 \times 12.00$ & $91 \times 3655$ \\
\hline C/2003 K4 (LINEAR) & 3131.342 & 2.61 & -20.34 & 2.37 & -43.12 & 4946 & $0.80 \times 11.00$ & $1375 \times 18908$ \\
\hline C/2003 K4 (LINEAR) & 3132.343 & 2.59 & -20.35 & 2.35 & -42.95 & 4380 & $0.60 \times 11.00$ & $1023 \times 18748$ \\
\hline C/2003 K4 (LINEAR) & 3329.344 & 1.20 & 14.81 & 1.51 & -28.23 & 1500 & $0.44 \times 12.00$ & $482 \times 13142$ \\
\hline 9P/Tempel 1 & 3553.955 & 1.51 & -0.21 & 0.89 & 8.95 & 7200 & $0.44 \times 12.00$ & $284 \times 7746$ \\
\hline 9P/Tempel 1 & 3554.954 & 1.51 & -0.15 & 0.89 & 9.07 & 7200 & $0.44 \times 12.00$ & $284 \times 7746$ \\
\hline 9P/Tempel 1 & 3555.955 & 1.51 & -0.04 & 0.90 & 9.19 & 7200 & $0.44 \times 12.00$ & $287 \times 7833$ \\
\hline 9P/Tempel 1 & 3557.007 & 1.51 & 0.09 & 0.90 & 9.48 & 9600 & $0.44 \times 12.00$ & $287 \times 7833$ \\
\hline 9P/Tempel 1 & 3557.955 & 1.51 & 0.20 & 0.91 & 9.44 & 7500 & $0.44 \times 12.00$ & $290 \times 7920$ \\
\hline 9P/Tempel 1 & 3558.952 & 1.51 & 0.31 & 0.91 & 9.55 & 7500 & $0.44 \times 12.00$ & $290 \times 7920$ \\
\hline 9P/Tempel 1 & 3559.954 & 1.51 & 0.43 & 0.92 & 9.68 & 7500 & $0.44 \times 12.00$ & $293 \times 8007$ \\
\hline 9P/Tempel 1 & 3560.952 & 1.51 & 0.55 & 0.93 & 9.80 & 7800 & $0.44 \times 12.00$ & $297 \times 8094$ \\
\hline 9P/Tempel 1 & 3561.953 & 1.51 & 0.66 & 0.93 & 9.91 & 7200 & $0.44 \times 12.00$ & $297 \times 8094$ \\
\hline 9P/Tempel 1 & 3562.956 & 1.51 & 0.78 & 0.94 & 10.04 & 7200 & $0.44 \times 12.00$ & $300 \times 8181$ \\
\hline 73P-C/SW 3 & 3882.367 & 0.95 & -4.17 & 0.15 & 12.31 & 4800 & $0.60 \times 12.00$ & $65 \times 1305$ \\
\hline 73P-B/SW 3 & 3898.369 & 0.94 & 1.79 & 0.25 & 13.10 & 4800 & $0.60 \times 12.00$ & $109 \times 2176$ \\
\hline 8P/Tuttle & 4481.021 & 1.04 & -4.29 & 0.36 & 21.64 & 3600 & $0.44 \times 10.00$ & $115 \times 2611$ \\
\hline 8P/Tuttle & 4493.018 & 1.03 & 0.40 & 0.52 & 24.72 & 3900 & $0.44 \times 10.00$ & $166 \times 3771$ \\
\hline 8P/Tuttle & 4500.017 & 1.03 & 3.16 & 0.62 & 24.16 & 3900 & $0.44 \times 10.00$ & $198 \times 4497$ \\
\hline 103P/Hartley 2 & 5505.288 & 1.06 & 2.53 & 0.16 & 7.08 & 2900 & $0.44 \times 12.00$ & $51 \times 1393$ \\
\hline 103P/Hartley 2 & 5505.328 & 1.06 & 2.55 & 0.16 & 7.19 & 3200 & $0.44 \times 12.00$ & $51 \times 1393$ \\
\hline 103P/Hartley 2 & 5510.287 & 1.07 & 4.05 & 0.18 & 7.96 & 2900 & $0.44 \times 12.00$ & $57 \times 1567$ \\
\hline 103P/Hartley 2 & 5510.328 & 1.07 & 4.07 & 0.18 & 8.07 & 3200 & $0.44 \times 12.00$ & $57 \times 1567$ \\
\hline 103P/Hartley 2 & 5511.363 & 1.08 & 4.37 & 0.19 & 8.27 & 900 & $0.44 \times 12.00$ & $60 \times 1654$ \\
\hline C/2009 P1 (Garradd) & 5692.383 & 3.25 & -16.91 & 3.50 & -44.66 & 3600 & $0.44 \times 12.00$ & $1117 \times 30461$ \\
\hline C/2009 P1 (Garradd) & 5727.322 & 2.90 & -16.89 & 2.57 & -46.38 & 3600 & $0.44 \times 12.00$ & $820 \times 22367$ \\
\hline C/2009 P1 (Garradd) & 5767.278 & 2.52 & -16.46 & 1.64 & -29.26 & 1800 & $0.44 \times 12.00$ & $523 \times 14273$ \\
\hline C/2009 P1 (Garradd) & 5813.991 & 2.09 & -14.82 & 1.47 & 14.79 & 4800 & $0.44 \times 12.00$ & $469 \times 12794$ \\
\hline C/2009 P1 (Garradd) & 5814.974 & 2.08 & -14.77 & 1.48 & 15.31 & 4800 & $0.44 \times 12.00$ & $472 \times 12881$ \\
\hline C/2009 P1 (Garradd) & 5815.982 & 2.07 & -14.71 & 1.49 & 15.84 & 4800 & $0.44 \times 12.00$ & $475 \times 12968$ \\
\hline
\end{tabular}

Notes. JD denotes Julian day, $r$ is the heliocentric distance, $\Delta$ the geocentric distance. $\dot{r}$ and $\dot{\Delta}$ are respectively the heliocentric and geocentric velocities. Exptime corresponds to the exposure time in seconds. Slit in arc seconds gives the size of the entrance slit of the spectrograph. The last column provides in $\mathrm{km}$ the spatial area covered by the slit.

\section{Discussion}

\subsection{The $G / R$ ratio at large heliocentric distance}

In Fig. 6, we noticed that $\mathrm{C} / 2001 \mathrm{Q} 4$ (NEAT) has a $\mathrm{G} / \mathrm{R}$ ratio of about 0.3 , so clearly much higher than other comets with values around 0.1. C/2001 Q4 was at 3.7 AU from the Sun, and this peculiarity led us to look more carefully at comets observed at large heliocentric distances (see Fig. 17 and Decock et al. 2011).

If we only consider the data taken at $r<2 \mathrm{AU}$, the $\mathrm{G} / \mathrm{R}$ ratio average value is equal to $0.09 \pm 0.02$, which is in good agreement with the ratio obtained by Bhardwaj \& Raghuram (2012) and Festou \& Feldman (1981) for $\mathrm{H}_{2} \mathrm{O}$ as the parent molecule (see Table 1). The high values of $\mathrm{G} / \mathrm{R}$ in $\mathrm{Q} 4$ (NEAT) at $3.7 \mathrm{AU}$ could be explained by the increasing contribution, at large heliocentric distances, of other parent molecules producing oxygen atoms. It is well known that the sublimation of $\mathrm{H}_{2} \mathrm{O}$ significantly decreases beyond $3 \mathrm{AU}$, while the sublimation of other ices like $\mathrm{CO}$ and/or $\mathrm{CO}_{2}$ dominates comet activity (i.e., Crovisier \& Encrenaz 2000). To investigate this hypothesis, we observed comet C/2009 P1 (Garradd) at four different heliocentric distances from 3.25 AU to 2.07 AU. Figure 18 presents the G/R intensity ratios as a function of $r$ for all spectra. A relation between the heliocentric distance and the $\mathrm{G} / \mathrm{R}$ intensity ratio can be seen: the latter is getting rapidly larger when the comet gets farther 


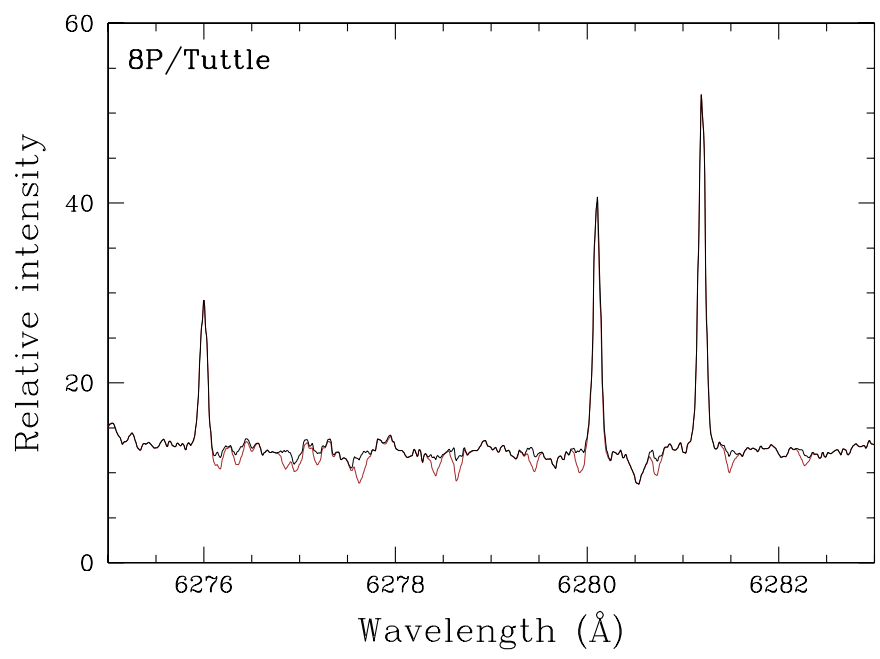

Fig. 3. The telluric absorption line correction in the vicinity of the red [OI] lines. The black spectrum has been corrected (thick line), the red one is uncorrected.

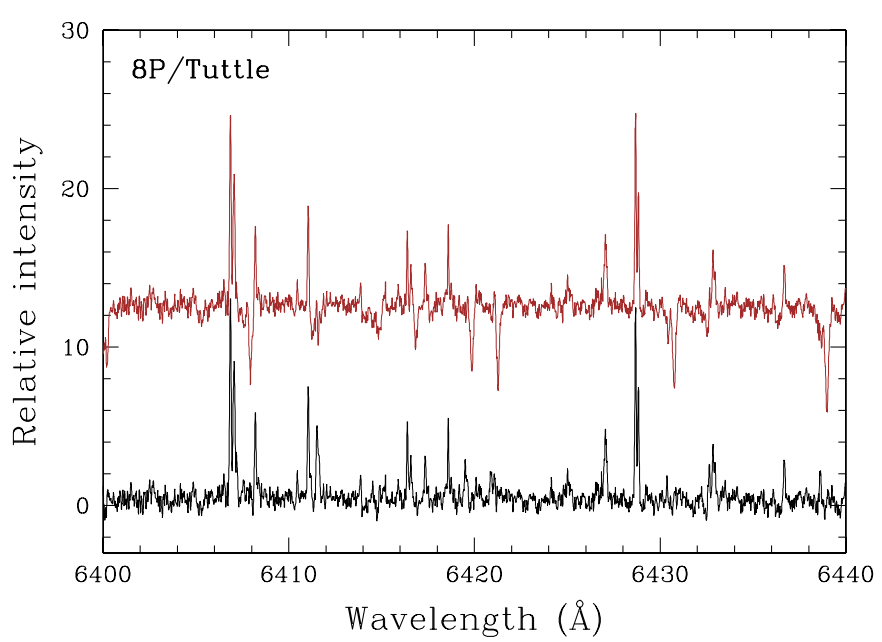

Fig. 4. Subtraction of the solar continuum. Upper spectrum (red) shows the spectrum of comet $8 \mathrm{P} /$ Tuttle not corrected for solar lines while in the lower one (black), solar continuum has been removed. Measurements of [OI] are made with the lower spectrum.

from the Sun. We added in this plot the Cochran \& Cochran (2001) and Cochran (2008) average values coming from their sample of eight comets. All these values are clumped around 1 AU with a ratio of $\sim 0.1 . \mathrm{C} / 2001 \mathrm{Q} 4$ is one of their eight comets and its $\mathrm{G} / \mathrm{R}$ ratio is $0.09 \pm 0.02$ at $r=0.98$ AU. A few other measurements found in the literature for comets observed at heliocentric distances beyond $2 \mathrm{AU}$ were also added to the graph. Furusho et al. (2006) used the Subaru telescope to analyze the forbidden oxygen lines in comet 116P/Wild 4 observed at $r=2.4$ AU. Capria et al. (2010) measured the intensity of these lines during and after the outburst of comet 17P/Holmes, which occurred on October 24, 2007 when the comet was located at about $r=2.5$ AU. McKay et al. (2012b) studied the [OI] lines of the two comets C/2006 W3 Christensen and C/2007 Q3 Siding Spring, respectively at 3.13 and 2.96 AU, with the ARCES echelle spectrometer of 3.5-m telescope at Apache Point Observatory. While the quality of the data is not as good, because the lines are faint and sometimes heavily blended with the telluric line, all these measurements show a relatively high value of

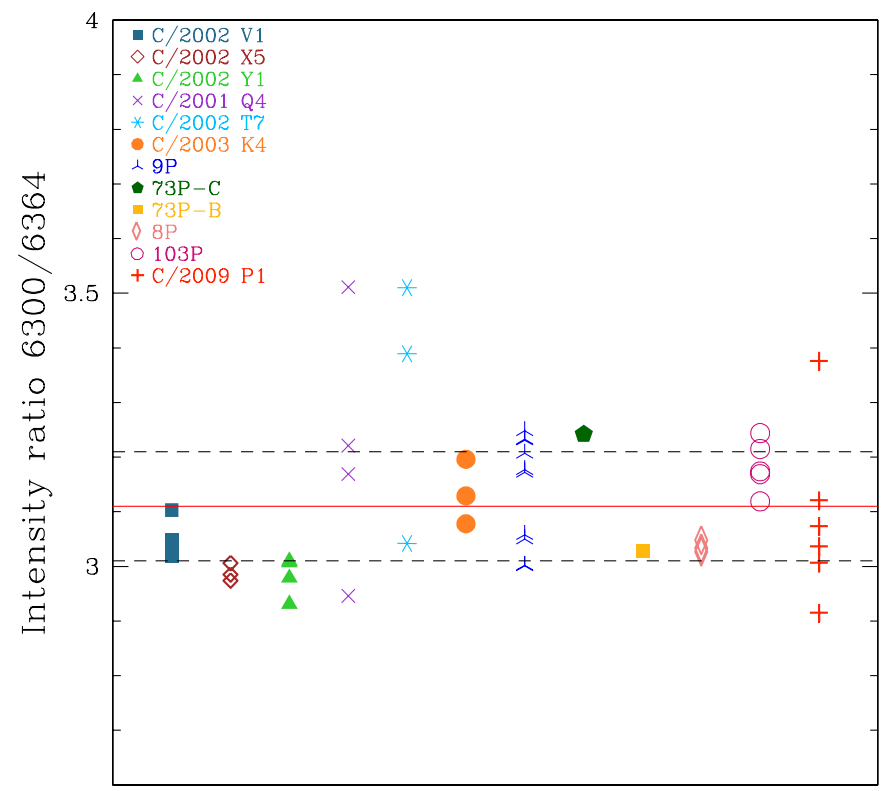

Fig. 5. The red doublet ratio for each comet. The spectra of a given comet have the same symbols. The average value of the sample $(3.11 \pm 0.10)$ is shown by the solid line. The dashed lines show the standard deviation $(\sigma)$.

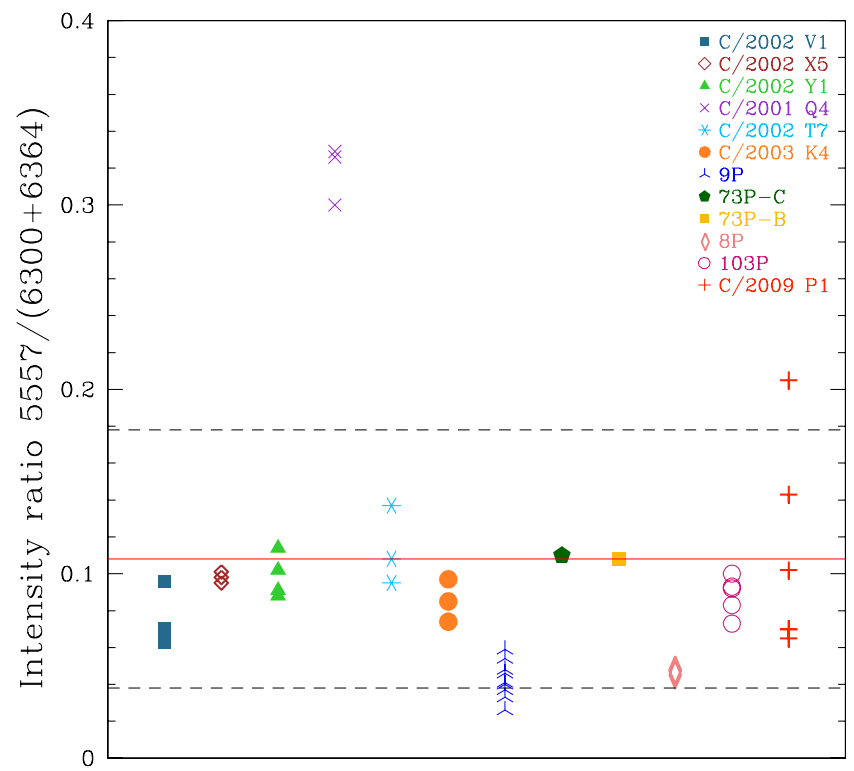

Fig. 6. Same as Fig. 5 for the G/R ratio. The mean value is $0.11 \pm 0.07$. Note the data points for comet Q4 (NEAT) at 3.7 AU.

the G/R ratio when $r>3$ AU. This confirms the hypothesis that oxygen is also coming from other molecules. Both $\mathrm{CO}$ and/or $\mathrm{CO}_{2}$ are obvious candidates as they produce high values of the $\mathrm{G} / \mathrm{R}$ ratio (see Table 1 ).

Using HST/STIS ${ }^{6}$ observations, Feldman et al. (2004) estimated at $4 \%$ the $\mathrm{CO}$ abundance relative to water in comet C/2001 Q4 located at 1 AU from the Sun. The study made by Biver et al. (2012) on comet C/2009 P1 provided an average value of $5 \%$ for the $\mathrm{CO}$ abundance when the comet was

6 HST/STIS is the acronym for Hubble Space Telescope/Space Telescope Imaging Spectrograph (http://wWw.stsci.edu/hst/ stis). 
A. Decock et al.: [OI] lines in comets at various heliocentric distances

Table 4. Intensities given in ADU (analog-to-digital units) and measured line ratios for the three forbidden oxygen lines in the spectra of each comet.

\begin{tabular}{|c|c|c|c|c|c|c|c|}
\hline \multirow[t]{2}{*}{ Comet } & \multirow[t]{2}{*}{ JD - 2450000.5} & \multirow[t]{2}{*}{$r(\mathrm{AU})$} & \multicolumn{3}{|c|}{ Intensity (ADU) } & \multirow{2}{*}{$\frac{I_{6300}}{I_{6364}}$} & \multirow{2}{*}{$\frac{I_{5577}}{I_{6300}+I_{6364}}$} \\
\hline & & & $5577.339 \AA$ & $6300.304 \AA$ & $6363.776 \AA$ & & \\
\hline C/2002 V1 (NEAT) & 2647.037 & 1.22 & 2348 & 27539 & 8875 & 3.10 & 0.065 \\
\hline C/2002 V1 (NEAT) & 2647.062 & 1.22 & 2255 & 26790 & 8875 & 3.02 & 0.063 \\
\hline C/2002 V1 (NEAT) & 2649.031 & 1.18 & 3220 & 35672 & 11704 & 3.05 & 0.068 \\
\hline C/2002 V1 (NEAT) & 2649.056 & 1.18 & 3137 & 33738 & 11105 & 3.04 & 0.070 \\
\hline C/2002 V1 (NEAT) & 2719.985 & 1.01 & 8657 & 68162 & 22381 & 3.05 & 0.096 \\
\hline C/2002 X5 (Kudo-Fujikawa) & 2705.017 & 1.06 & 4016 & 31491 & 10589 & 2.97 & 0.095 \\
\hline C/2002 X5 (Kudo-Fujikawa) & 2705.039 & 1.07 & 4430 & 32822 & 10997 & 2.98 & 0.101 \\
\hline C/2002 X5 (Kudo-Fujikawa) & 2705.060 & 1.07 & 4249 & 32510 & 10816 & 3.01 & 0.098 \\
\hline C/2002 Y1 (Juels-Holvorcem) & 2788.395 & 1.14 & 6223 & 50960 & 17387 & 2.93 & 0.091 \\
\hline C/2002 Y1 (Juels-Holvorcem) & 2788.416 & 1.14 & 6386 & 54683 & 18167 & 3.01 & 0.088 \\
\hline C/2002 Y1 (Juels-Holvorcem) & 2789.394 & 1.16 & 3942 & 25792 & 8659 & 2.98 & 0.114 \\
\hline C/2002 Y1 (Juels-Holvorcem) & 2789.415 & 1.16 & 4254 & 31262 & 10398 & 3.01 & 0.102 \\
\hline C/2001 Q4 (NEAT) & 2883.293 & 3.73 & 143 & 324 & 110 & 2.95 & 0.329 \\
\hline C/2001 Q4 (NEAT) & 2883.349 & 3.73 & 151 & 353 & 110 & 3.22 & 0.326 \\
\hline C/2001 Q4 (NEAT) & 2889.236 & 3.67 & - & 354 & 112 & 3.17 & - \\
\hline C/2001 Q4 (NEAT) & 2889.320 & 3.66 & 261 & 677 & 193 & 3.51 & 0.300 \\
\hline C/2002 T7 (LINEAR) & 3131.421 & 0.68 & 116522 & 642304 & 211120 & 3.04 & 0.137 \\
\hline C/2002 T7 (LINEAR) & 3151.976 & 0.94 & 50544 & 365664 & 104187 & 3.51 & 0.108 \\
\hline C/2002 T7 (LINEAR) & 3152.036 & 0.94 & 40165 & 327184 & 96554 & 3.39 & 0.095 \\
\hline C/2003 K4 (LINEAR) & 3131.342 & 2.61 & 464 & 3609 & 1172 & 3.08 & 0.097 \\
\hline C/2003 K4 (LINEAR) & 3132.343 & 2.59 & 561 & 5027 & 1607 & 3.13 & 0.085 \\
\hline C/2003 K4 (LINEAR) & 3329.344 & 1.20 & 9244 & 94723 & 29640 & 3.20 & 0.074 \\
\hline 9P/Tempel 1 & 3553.955 & 1.51 & 230 & 3178 & 1039 & 3.06 & 0.054 \\
\hline 9P/Tempel 1 & 3554.954 & 1.51 & 172 & 3153 & 1051 & 3.00 & 0.041 \\
\hline 9P/Tempel 1 & 3555.955 & 1.51 & - & 3894 & 1297 & 3.00 & - \\
\hline 9P/Tempel 1 & 3557.007 & 1.51 & 341 & 5618 & 1842 & 3.05 & 0.046 \\
\hline 9P/Tempel 1 & 3557.955 & 1.51 & 223 & 5154 & 1595 & 3.23 & 0.033 \\
\hline 9P/Tempel 1 & 3558.952 & 1.51 & 277 & 5267 & 1629 & 3.23 & 0.040 \\
\hline 9P/Tempel 1 & 3559.954 & 1.51 & 291 & 4618 & 1440 & 3.21 & 0.048 \\
\hline 9P/Tempel 1 & 3560.952 & 1.51 & 388 & 4984 & 1571 & 3.17 & 0.059 \\
\hline 9P/Tempel 1 & 3561.953 & 1.51 & 211 & 4281 & 1347 & 3.18 & 0.037 \\
\hline 9P/Tempel 1 & 3562.956 & 1.51 & 142 & 4162 & 1281 & 3.25 & 0.026 \\
\hline 73P-C/Schwassmann-Wachmann 3 & 3882.367 & 0.95 & 16505 & 115170 & 35526 & 3.24 & 0.110 \\
\hline 73P-B/Schwassmann-Wachmann 3 & 3898.369 & 0.94 & 4890 & 33987 & 11226 & 3.03 & 0.108 \\
\hline 8P/Tuttle & 4481.021 & 1.04 & 1779 & 28517 & 9385 & 3.04 & 0.047 \\
\hline 8P/Tuttle & 4493.018 & 1.03 & 4437 & 74360 & 24565 & 3.03 & 0.045 \\
\hline 8P/Tuttle & 4500.017 & 1.03 & 4179 & 64979 & 21424 & 3.03 & 0.048 \\
\hline 103P/Hartley 2 & 5505.288 & 1.06 & 45 & 373 & 120 & 3.12 & 0.092 \\
\hline 103P/Hartley 2 & 5505.328 & 1.06 & 50 & 456 & 144 & 3.22 & 0.100 \\
\hline 103P/Hartley 2 & 5510.287 & 1.07 & 51 & 390 & 121 & 3.24 & 0.073 \\
\hline 103P/Hartley 2 & 5510.328 & 1.07 & 41 & 425 & 131 & 3.17 & 0.083 \\
\hline 103P/Hartley 2 & 5511.363 & 1.08 & 48 & 395 & 125 & 3.17 & 0.093 \\
\hline C/2009 P1 (Garradd) & 5692.383 & 3.25 & 180 & 652 & 224 & 2.92 & 0.205 \\
\hline C/2009 P1 (Garradd) & 5727.322 & 2.90 & 129 & 693 & 205 & 3.38 & 0.143 \\
\hline C/2009 P1 (Garradd) & 5767.278 & 2.52 & 1110 & 8276 & 2652 & 3.12 & 0.102 \\
\hline C/2009 P1 (Garradd) & 5813.991 & 2.09 & 1594 & 17035 & 5666 & 3.01 & 0.070 \\
\hline C/2009 P1 (Garradd) & 5814.974 & 2.08 & 1571 & 16873 & 5489 & 3.07 & 0.070 \\
\hline C/2009 P1 (Garradd) & 5815.982 & 2.07 & 1506 & 17557 & 5780 & 3.04 & 0.065 \\
\hline
\end{tabular}

Notes. For the third spectrum of comets C/2001 Q4 and 9P, the green line could not be measured because of a contamination by a cosmic ray.

at 1.9 $\mathrm{AU}$ at pre-perihelion phase (October 2011). At large heliocentric distances, $\mathrm{CO} / \mathrm{H}_{2} \mathrm{O}$ had to be even higher, so the $\mathrm{CO}$ molecule could be a candidate to explain such a high $\mathrm{G} / \mathrm{R}$ ratio. However, Bhardwaj \& Raghuram (2012) show that after the photodissociation of $\mathrm{H}_{2} \mathrm{O}$, the next main source for the green line emission is $\mathrm{CO}_{2}$ with $10-40 \%$ abundance relative to water. They compared their model with the observational values obtained for C/1996 B2 (Hyakutake) by Morrison et al. (1997) and Cochran (2008) when the comet was around 1 AU. Hyakutake is also rich in $\mathrm{CO}$, and its abundance was evaluated at $22 \%$ at $1 \mathrm{AU}$ (Biver et al. 1999). Assuming a $\mathrm{CO}_{2}$ abundance of $1 \%$ and an $\mathrm{O}\left({ }^{1} \mathrm{~S}\right)$ yield of $0.2 \%$, Bhardwaj \& Raghuram (2012) concludes that the production rates of the $\mathrm{O}\left({ }^{1} \mathrm{~S}\right)$ are similar for $\mathrm{CO}$ and $\mathrm{CO}_{2}(\sim 10-25 \%)$ with such abundances. Therefore, since C/2001Q4 and C/2009 P1 have less CO than Hyakutake, $\mathrm{CO}_{2}$ molecules could instead be the main contributor to the formation of the green line emission for these two comets observed at large heliocentric distance. The $\mathrm{CO}_{2}$ measurements are unfortunately difficult to obtain to confirm this hypothesis. If this is the case, estimated $\mathrm{G} / \mathrm{R}$ ratios might be used as a proxy for the $\mathrm{CO}_{2}$ relative abundance, but such a relation should be carefully calibrated on a sample of comets with known $\mathrm{CO}_{2}$. 
Table 5. Average values of the line ratios for each comet.

\begin{tabular}{lccc}
\hline \hline Comet & $N$ & $\frac{I_{6300}}{I_{6364}}$ & $\frac{I_{5577}}{I_{6300}+I_{6364}}$ \\
\hline C/2002 V1 (NEAT) & 5 & $3.05 \pm 0.03$ & $0.07 \pm 0.01$ \\
C/2002 X5 (Kudo-Fujikawa) & 3 & $2.99 \pm 0.02$ & $0.10 \pm 0.003$ \\
C/2002 Y1 (Juels-Holvorcem) & 4 & $2.98 \pm 0.04$ & $0.10 \pm 0.01$ \\
C/2001 Q4 (NEAT) & 4 & $3.21 \pm 0.23$ & $0.32 \pm 0.02$ \\
C/2002 T7 (LINEAR) & 3 & $3.31 \pm 0.18$ & $0.15 \pm 0.05$ \\
C/2003 K4 (LINEAR) & 3 & $3.14 \pm 0.06$ & $0.09 \pm 0.01$ \\
9P/Tempel 1 & 10 & $3.14 \pm 0.10$ & $0.04 \pm 0.01$ \\
73P-C/SW 3 & 1 & 3.25 & 0.11 \\
73P-B/SW 3 & 1 & 3.03 & 0.11 \\
8P/Tuttle & 3 & $3.03 \pm 0.01$ & $0.05 \pm 0.01$ \\
103P/Hartley 2 & 5 & $3.15 \pm 0.07$ & $0.08 \pm 0.02$ \\
C/2009 P1 (Garradd) & 2 & $3.15 \pm 0.33$ & $0.017 \pm 0.04$ \\
C/2009 P1 (Garradd) & 3 & $3.04 \pm 0.03$ & $0.07 \pm 0.003$ \\
\hline
\end{tabular}

Notes. The errors listed are the standard deviation of the $\mathrm{N}$ spectra available for each comet. 73P-C/SW3 and 73P-C/SW3 have no error because we obtained only one spectrum for these comets.

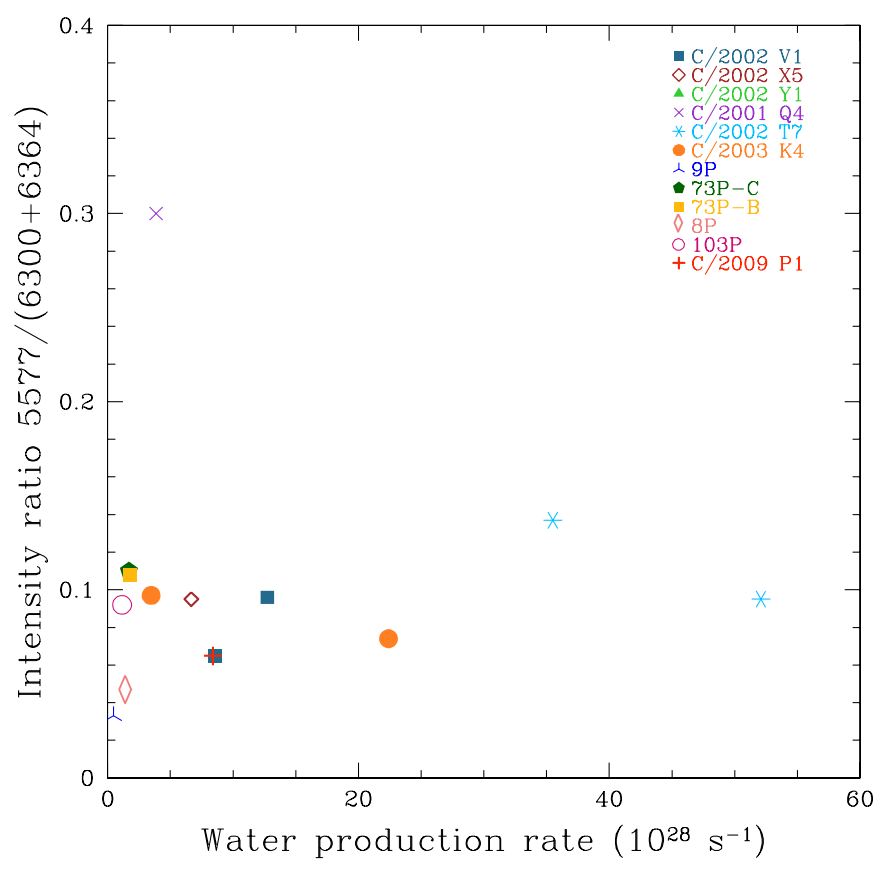

Fig. 7. The G/R ratio against the water production rate.

Using Eq. (8) and only considering the main parent species of oxygen atoms (i.e $\mathrm{H}_{2} \mathrm{O}, \mathrm{CO}_{2}$, and $\mathrm{CO}$ ), we can write the $\mathrm{G} / \mathrm{R}$ ratio as

$\mathrm{G} / \mathrm{R}=\frac{I^{\text {green }}}{I^{\text {red }}}=\frac{W_{\mathrm{H}_{2} \mathrm{O}}^{\text {green }} \beta^{\text {green }} N_{\mathrm{H}_{2} \mathrm{O}}+W_{\mathrm{CO}_{2}}^{\text {green }} \beta^{\text {green }} N_{\mathrm{CO}_{2}}+W_{\mathrm{CO}}^{\text {green }} \beta^{\text {green }} N_{\mathrm{CO}}}{W_{\mathrm{H}_{2} \mathrm{O}}^{\text {red }} \beta^{\text {red }} N_{\mathrm{H}_{2} \mathrm{O}}+W_{\mathrm{CO}_{2}}^{\text {red }} \beta^{\text {red }} N_{\mathrm{CO}_{2}}+W_{\mathrm{CO}}^{\text {red }} \beta^{\text {red }} N_{\mathrm{CO}}}$,

where $W_{b}^{a}=\alpha_{b}^{a} \tau_{b}^{-1}$ corresponds to the effective production rates of the $\mathrm{O}\left({ }^{1} \mathrm{~S}\right)$ and $\mathrm{O}\left({ }^{1} \mathrm{D}\right)$ states ${ }^{7}$. However, if we consider that oxygen atoms only come from $\mathrm{H}_{2} \mathrm{O}$ and $\mathrm{CO}$ molecules, we found that $\mathrm{G} / \mathrm{R}$ ratio does not change strongly: it varies from 0.08 to 0.1 for a CO abundance between $10 \%$ and $80 \%$. Therefore, we

\footnotetext{
7 The production rates $W_{b}^{a}$ are given in Tables 1 and 2 of Bhardwaj \& Raghuram (2012) (i.e $W_{\mathrm{H}_{2} \mathrm{O}}^{\text {red }}=8 \times 10^{-7} \mathrm{~s}^{-1}, W_{\mathrm{H}_{2} \mathrm{O}}^{\text {green }}=6.4 \times 10^{-8} \mathrm{~s}^{-1}$, $W_{\mathrm{CO}_{2}}^{\text {green }}=7.2 \times 10^{-7} \mathrm{~s}^{-1}, W_{\mathrm{CO}_{2}}^{\text {red }}=1.2 \times 10^{-6} \mathrm{~s}^{-1}, W_{\mathrm{CO}}^{\text {green }}=4 \times 10^{-8} \mathrm{~s}^{-1}$ and $W_{\mathrm{CO}}^{\text {red }}=5.1 \times 10^{-8} \mathrm{~s}^{-1}$ ) and $\beta^{\text {green }}=0.91$ (Slanger et al. 2006).
}

assume that the [OI] atoms are only produced by the $\mathrm{H}_{2} \mathrm{O}$ and $\mathrm{CO}_{2}$ photodissociations. Equation (10) can thus be simplified as

$\mathrm{G} / \mathrm{R}=\frac{I^{\text {green }}}{I^{\text {red }}}=\frac{W_{\mathrm{H}_{2} \mathrm{O}}^{\text {green }} \beta^{\text {green }} N_{\mathrm{H}_{2} \mathrm{O}}+W_{\mathrm{CO}_{2}}^{\text {green }} \beta^{\text {green }} N_{\mathrm{CO}_{2}}}{W_{\mathrm{H}_{2} \mathrm{O}}^{\text {red }} \beta^{\text {red }} N_{\mathrm{H}_{2} \mathrm{O}}+W_{\mathrm{CO}_{2}}^{\text {red }} \beta^{\text {red }} N_{\mathrm{CO}_{2}}}$.

Therefore, the $\mathrm{CO}_{2} / \mathrm{H}_{2} \mathrm{O}$ abundance can be computed by

$\frac{N_{\mathrm{CO}_{2}}}{N_{\mathrm{H}_{2} \mathrm{O}}}=\frac{(\mathrm{G} / \mathrm{R}) W_{\mathrm{H}_{2} \mathrm{O}}^{\text {red }}-\beta^{\text {green }} W_{\mathrm{H}_{2} \mathrm{O}}^{\text {green }}}{\beta^{\text {green }} W_{\mathrm{CO}_{2}}^{\text {green }}-(G / R) W_{\mathrm{CO}_{2}}^{\text {red }}}$.

The $W_{b}^{a}$ are independent of the heliocentric distance because all of them depend on the same heliocentric distance. This is also the case for the values given in Table 1. Considering the same values for $W_{b}^{a}$ and $\beta^{\text {green }}$, we also computed the $\mathrm{CO}_{2} / \mathrm{H}_{2} \mathrm{O}$ abundance from the $\mathrm{G} / \mathrm{R}$ ratios of comets $\mathrm{C} / 2001 \mathrm{Q} 4$ and $\mathrm{C} / 2009 \mathrm{P} 1$ measured by Cochran (2008) and McKay et al. (2012a), respectively. These results are shown in Fig. 19. We noticed that $\mathrm{CO}_{2} / \mathrm{H}_{2} \mathrm{O}$ is as high as $75 \%$ in $\mathrm{C} / 2001 \mathrm{Q} 4$ and that $\mathrm{CO}_{2}$ starts to contribute to the $\mathrm{G} / \mathrm{R}$ ratio at $2.5 \mathrm{AU}$ in the comets. This heliocentric distance limit is also shown by Ootsubo et al. (2012) from a sample of 17 comets observed with the AKARI IR space telescope.

Therefore, the forbidden oxygen line measurement could become a new way to determine the $\mathrm{CO}_{2}$ abundance in comets at different heliocentric distances from ground observations, while the direct measurement of $\mathrm{CO}_{2}$ molecules is only possible from space.

\subsection{The [OI] lines widths}

One explanation for the large width of the [OI] green line could be that exciting photons producing oxygen atoms in the ${ }^{1} \mathrm{~S}$ state have more energy than the Lyman $\alpha$ photons and/or come from another parent molecule. Indeed, Fig. 7 of Bhardwaj \& Raghuram (2012) shows that the wavelength region for the major production of $\mathrm{O}\left({ }^{1} \mathrm{~S}\right)$ coming from $\mathrm{CO}_{2}$ seems to be the 955-1165 $\AA$ band. New theoretical studies could be necessary to evaluate the excess velocity for the $\mathrm{O}\left({ }^{1} \mathrm{~S}\right)$ state coming from the photodissociations of both $\mathrm{H}_{2} \mathrm{O}$ and $\mathrm{CO}_{2}$.

In Fig. 12, we also notice that the [OI] red lines of the comet $\mathrm{C} / 2001 \mathrm{Q} 4$ are wider than in other comets, while the width of the green line is normal compared to other comets. This peculiarity seems real as both red lines show the same width, and they are 2- $\sigma$ away from the average value of the sample $\left(1.58 \pm 0.30 \mathrm{~km} \mathrm{~s}^{-1}\right)$. The telluric lines in $\mathrm{C} / 2001 \mathrm{Q} 4$ have the usual width $\left(0.99 \pm 0.10 \mathrm{~km} \mathrm{~s}^{-1}\right.$ for the $6300 \AA$ line, $0.87 \pm 0.04 \mathrm{~km} \mathrm{~s}^{-1}$ for the $6364 \AA$ line and $0.80 \pm 0.26 \mathrm{~km} \mathrm{~s}^{-1}$ for the green line), excluding a problem with the spectra or the analysis. The width of the red lines and the green line is similar in only this case $\left(2.57 \pm 0.16 \mathrm{~km} \mathrm{~s}^{-1}\right.$ for the $6300 \AA$ line, $2.49 \pm 0.15 \mathrm{~km} \mathrm{~s}^{-1}$ for the $6364 \AA$ and $2.46 \pm 0.13 \mathrm{~km} \mathrm{~s}^{-1}$ for the $5577 \AA$ line) and could give us clues to the process in play. As previously discussed, at large heliocentric distances, the [OI] lines could mainly come from the $\mathrm{CO}_{2}$ molecules which mainly photo-dissociate in the ${ }^{1} \mathrm{~S}$ state, while at low heliocentric distances, they essentially come from $\mathrm{H}_{2} \mathrm{O}$, which primarily dissociates in the ${ }^{1} \mathrm{D}$ state (Table 1 ). This means that at large distances, the red lines are mainly produced through the ${ }^{1} \mathrm{~S}-{ }^{1} \mathrm{D}$ channel, together with the green line, while at low distances they are mostly produced directly from the ${ }^{1} \mathrm{D}$ state since $\mathrm{H}_{2} \mathrm{O}$ dominates. We thus expect the widths of the red and green lines to be similar at high distances because the $\mathrm{O}\left({ }^{1} \mathrm{~S}\right)$ and 
A. Decock et al.: [OI] lines in comets at various heliocentric distances

Table 6. Measured observed FWHM $(\AA)$ and intrinsic velocity widths $\left(\mathrm{km} \mathrm{s}^{-1}\right)$ for the three forbidden oxygen lines in the spectra of each comet.

\begin{tabular}{|c|c|c|c|c|c|c|c|c|}
\hline \multirow[t]{2}{*}{ Comet } & \multirow{2}{*}{\multicolumn{2}{|c|}{$\mathrm{JD}-2450000.5 r(\mathrm{AU})$}} & \multicolumn{3}{|c|}{$F W H M_{\text {observed }}(\AA)$} & \multicolumn{3}{|c|}{$F W H M_{\text {intrinsic }}\left(\mathrm{km} \mathrm{s}^{-1}\right)$} \\
\hline & & & $5577.339 \AA$ & $\AA 6300.304 \AA$ & $6363.776 \AA$ & $5577.339 \AA$ & $6300.304 \AA$ & $6363.776 \AA$ \\
\hline $\mathrm{C} / 2002$ V1 (NEAT) & 2647.037 & 1.22 & 0.092 & 0.098 & 0.094 & 2.08 & 1.57 & 1.40 \\
\hline C/2002 V1 (NEAT) & 2647.062 & 1.22 & 0.089 & 0.095 & 0.095 & 1.95 & 1.42 & 1.44 \\
\hline C/2002 V1 (NEAT) & 2649.031 & 1.18 & 0.096 & 0.096 & 0.095 & 2.23 & 1.45 & 1.42 \\
\hline C/2002 V1 (NEAT) & 2649.056 & 1.18 & 0.093 & 0.095 & 0.094 & 2.11 & 1.40 & 1.38 \\
\hline C/2002 V1 (NEAT) & 2719.985 & 1.01 & 0.108 & 0.098 & 0.096 & 2.76 & 1.54 & 1.49 \\
\hline C/2002 X5 (Kudo-Fujikawa) & 2705.017 & 1.06 & 0.104 & 0.094 & 0.093 & 2.64 & 1.46 & 1.50 \\
\hline C/2002 X5 (Kudo-Fujikawa) & 2705.039 & 1.07 & 0.104 & 0.095 & 0.095 & 2.61 & 1.54 & 1.60 \\
\hline C/2002 X5 (Kudo-Fujikawa) & 2705.060 & 1.07 & 0.104 & 0.095 & 0.094 & 2.64 & 1.50 & 1.54 \\
\hline C/2002 Y1 (Juels-Holvorcem) & 2788.395 & 1.14 & 0.106 & 0.092 & 0.091 & 2.88 & 1.52 & 1.47 \\
\hline C/2002 Y1 (Juels-Holvorcem) & 2788.416 & 1.14 & 0.103 & 0.092 & 0.091 & 2.75 & 1.54 & 1.49 \\
\hline C/2002 Y1 (Juels-Holvorcem) & 2789.394 & 1.16 & 0.113 & 0.095 & 0.094 & 3.09 & 1.59 & 1.57 \\
\hline C/2002 Y1 (Juels-Holvorcem) & 2789.415 & 1.16 & 0.112 & 0.095 & 0.094 & 3.03 & 1.61 & 1.59 \\
\hline C/2001 Q4 (NEAT) & 2883.293 & 3.73 & 0.101 & 0.116 & 0.117 & 2.51 & 2.39 & 2.39 \\
\hline C/2001 Q4 (NEAT) & 2883.349 & 3.73 & 0.097 & 0.123 & 0.125 & 2.31 & 2.63 & 2.69 \\
\hline C/2001 Q4 (NEAT) & 2889.236 & 3.67 & - & 0.119 & 0.121 & - & 2.49 & 2.52 \\
\hline C/2001 Q4 (NEAT) & 2889.320 & 3.66 & 0.102 & 0.125 & 0.117 & 2.55 & 2.75 & 2.36 \\
\hline C/2002 T7 (LINEAR) & 3131.421 & 0.68 & 0.115 & 0.099 & 0.100 & 3.12 & 1.82 & 1.80 \\
\hline C/2002 T7 (LINEAR) & 3151.976 & 0.94 & 0.101 & 0.089 & 0.090 & 2.84 & 1.70 & 1.71 \\
\hline C/2002 T7 (LINEAR) & 3152.036 & 0.94 & 0.100 & 0.088 & 0.090 & 2.81 & 1.66 & 1.72 \\
\hline C/2003 K4 (LINEAR) & 3131.342 & 2.61 & 0.131 & 0.135 & 0.136 & 2.51 & 1.81 & 1.66 \\
\hline C/2003 K4 (LINEAR) & 3132.343 & 2.59 & 0.106 & 0.108 & 0.105 & 2.76 & 2.12 & 1.94 \\
\hline C/2003 K4 (LINEAR) & 3329.344 & 1.20 & 0.097 & 0.094 & 0.096 & 2.38 & 1.52 & 1.52 \\
\hline 9P/Tempel 1 & 3553.955 & 1.51 & 0.110 & 0.096 & 0.093 & 2.91 & 1.61 & 1.43 \\
\hline 9P/Tempel 1 & 3554.954 & 1.51 & 0.091 & 0.095 & 0.094 & 2.14 & 1.59 & 1.48 \\
\hline 9P/Tempel 1 & 3555.955 & 1.51 & - & 0.094 & 0.094 & - & 1.56 & 1.46 \\
\hline 9P/Tempel 1 & 3557.007 & 1.51 & 0.091 & 0.095 & 0.095 & 2.03 & 1.55 & 1.44 \\
\hline 9P/Tempel 1 & 3557.955 & 1.51 & 0.084 & 0.095 & 0.095 & 1.75 & 1.54 & 1.47 \\
\hline 9P/Tempel 1 & 3558.952 & 1.51 & 0.084 & 0.095 & 0.095 & 1.79 & 1.58 & 1.48 \\
\hline 9P/Tempel 1 & 3559.954 & 1.51 & 0.101 & 0.101 & 0.097 & 2.57 & 1.84 & 1.60 \\
\hline 9P/Tempel 1 & 3560.952 & 1.51 & 0.106 & 0.098 & 0.096 & 2.77 & 1.72 & 1.52 \\
\hline 9P/Tempel 1 & 3561.953 & 1.51 & 0.087 & 0.096 & 0.095 & 1.92 & 1.63 & 1.48 \\
\hline 9P/Tempel 1 & 3562.956 & 1.51 & 0.081 & 0.096 & 0.095 & 1.67 & 1.61 & 1.48 \\
\hline 73P-C/Schwassmann-Wachmann 3 & 3882.367 & 0.95 & 0.104 & 0.106 & 0.107 & 2.12 & 1.31 & 1.30 \\
\hline 73P-B/Schwassmann-Wachmann 3 & 3898.369 & 0.94 & 0.110 & 0.108 & 0.108 & 2.46 & 1.54 & 1.48 \\
\hline 8P/Tuttle & 4481.021 & 1.04 & 0.091 & 0.093 & 0.091 & 2.16 & 1.55 & 1.36 \\
\hline 8P/Tuttle & 4493.018 & 1.03 & 0.093 & 0.093 & 0.092 & 2.21 & 1.55 & 1.41 \\
\hline 8P/Tuttle & 4500.017 & 1.03 & 0.095 & 0.093 & 0.091 & 2.28 & 1.47 & 1.33 \\
\hline 103P/Hartley 2 & 5505.288 & 1.06 & 0.095 & 0.091 & 0.097 & 2.30 & 1.35 & 1.29 \\
\hline 103P/Hartley 2 & 5505.328 & 1.06 & 0.090 & 0.082 & 0.081 & 2.31 & 1.26 & 1.25 \\
\hline 103P/Hartley 2 & 5510.287 & 1.07 & 0.092 & 0.081 & 0.083 & 2.31 & 1.26 & 1.06 \\
\hline 103P/Hartley 2 & 5510.328 & 1.07 & 0.092 & 0.081 & 0.079 & 2.24 & 1.27 & 1.17 \\
\hline 103P/Hartley 2 & 5511.363 & 1.08 & 0.090 & 0.081 & 0.081 & 2.19 & 1.23 & 1.18 \\
\hline C/2009 P1 (Garradd) & 5692.383 & 3.25 & 0.095 & 0.091 & 0.097 & 2.28 & 1.67 & 1.87 \\
\hline C/2009 P1 (Garradd) & 5727.322 & 2.90 & 0.099 & 0.083 & 0.089 & 2.54 & 1.27 & 1.49 \\
\hline C/2009 P1 (Garradd) & 5767.278 & 2.52 & 0.090 & 0.083 & 0.084 & 2.19 & 1.30 & 1.31 \\
\hline C/2009 P1 (Garradd) & 5813.991 & 2.09 & 0.090 & 0.085 & 0.087 & 2.18 & 1.25 & 1.33 \\
\hline C/2009 P1 (Garradd) & 5814.974 & 2.08 & 0.089 & 0.086 & 0.087 & 2.16 & 1.33 & 1.33 \\
\hline C/2009 P1 (Garradd) & 5815.982 & 2.07 & 0.090 & 0.087 & 0.089 & 2.21 & 1.57 & 1.58 \\
\hline
\end{tabular}

Notes. For the third spectrum of comets C/2001 Q4 and 9P, the green line could not be used because of the contamination by a strong cosmic ray.

$\mathrm{O}\left({ }^{1} \mathrm{D}\right)$ atoms are mostly produced from a same molecule, while they can differ at low distances, as observed. $\mathrm{CO}_{2}$ is the best candidate, and the larger width could be explained by the main excitation source of $\mathrm{CO}_{2}$, the 955-1165 $\AA$ band, which is more energetic than Ly- $\alpha$ photons, as shown in Fig. 11 of Bhardwaj \& Raghuram (2012). This particularity is not seen for comet C/2009 P1 (Garradd), maybe because the spectrum is not taken at a sufficiently large heliocentric distance, and the $\mathrm{CO}_{2}$ abundance was lower than in C/2001 Q4 (NEAT). Either way, this hypothesis needs to be confirmed by observing other comets at large heliocentric distances (>3.5 AU).

\section{3. $103 P /$ Hartley 2}

The Jupiter family comet 103P/Hartley 2 has been found to be poor in $\mathrm{CO}$ (Weaver et al. 2011) and rich in $\mathrm{CO}_{2}$ with an $\mathrm{CO}_{2}$ abundance of $\sim 20 \%$ relative to the water from EPOXI measurements (A'Hearn et al. 2011) and with an abundance of $\sim 10 \%$ from the ISO observations (Crovisier et al. 1999). Thanks to Eq. (12), we could evaluate the $\mathrm{CO}_{2}$ abundances from our $\mathrm{G} / \mathrm{R}$ ratios measured at $\sim 1 \mathrm{AU}$ and compare them with the values obtained by EPOXI and ISO observations. The results are given in Table 9. The mean $\mathrm{G} / \mathrm{R}$ ratio of $0.09 \pm 0.01$ is similar 
Table 7. Average values of the intrinsic line width for each comet.

\begin{tabular}{lcccc}
\hline \hline \multirow{2}{*}{ Comet } & $N$ & \multicolumn{3}{c}{$F W H M_{\text {intrinsic }}\left(\mathrm{km} \mathrm{s}^{-1}\right)$} \\
& & $5577.339 \AA$ & $6300.304 \AA$ & $6363.776 \AA$ \\
\hline C/2002 V1 & 5 & $1.48 \pm 0.07$ & $1.43 \pm 0.04$ & $2.22 \pm 0.32$ \\
C/2002 X5 & 3 & $1.48 \pm 0.07$ & $1.43 \pm 0.04$ & $2.22 \pm 0.32$ \\
C/2002 Y1 & 4 & $1.57 \pm 0.04$ & $1.53 \pm 0.06$ & $2.94 \pm 0.15$ \\
C/2001 Q4 & 4 & $2.57 \pm 0.16$ & $2.49 \pm 0.15$ & $2.46 \pm 0.13$ \\
C/2002 T7 & 3 & $1.77 \pm 0.09$ & $1.72 \pm 0.07$ & $2.47 \pm 0.64$ \\
C/2003 K4 & 3 & $1.82 \pm 0.30$ & $1.71 \pm 0.21$ & $2.55 \pm 0.19$ \\
9P & 10 & $1.62 \pm 0.09$ & $1.48 \pm 0.05$ & $2.23 \pm 0.47$ \\
73P-C & 1 & 1.27 & 1.27 & 2.13 \\
73P-B & 1 & 1.54 & 1.47 & 2.56 \\
8P & 3 & $1.53 \pm 0.05$ & $1.37 \pm 0.04$ & $2.22 \pm 0.06$ \\
103P & 5 & $1.28 \pm 0.05$ & $2.00 \pm 0.07$ & $2.15 \pm 0.20$ \\
C/2009 P1 & 2 & $1.47 \pm 0.29$ & $1.68 \pm 0.27$ & $2.41 \pm 0.19$ \\
C/2009 P1 & 3 & $1.34 \pm 0.17$ & $1.42 \pm 0.15$ & $2.18 \pm 0.02$ \\
\hline
\end{tabular}

Notes. The errors listed are the standard deviation of the $N$ spectra available for each comet. 73P-C/SW3 and 73P-C/SW3 have no error quoted because we obtained only one spectrum for these comets.

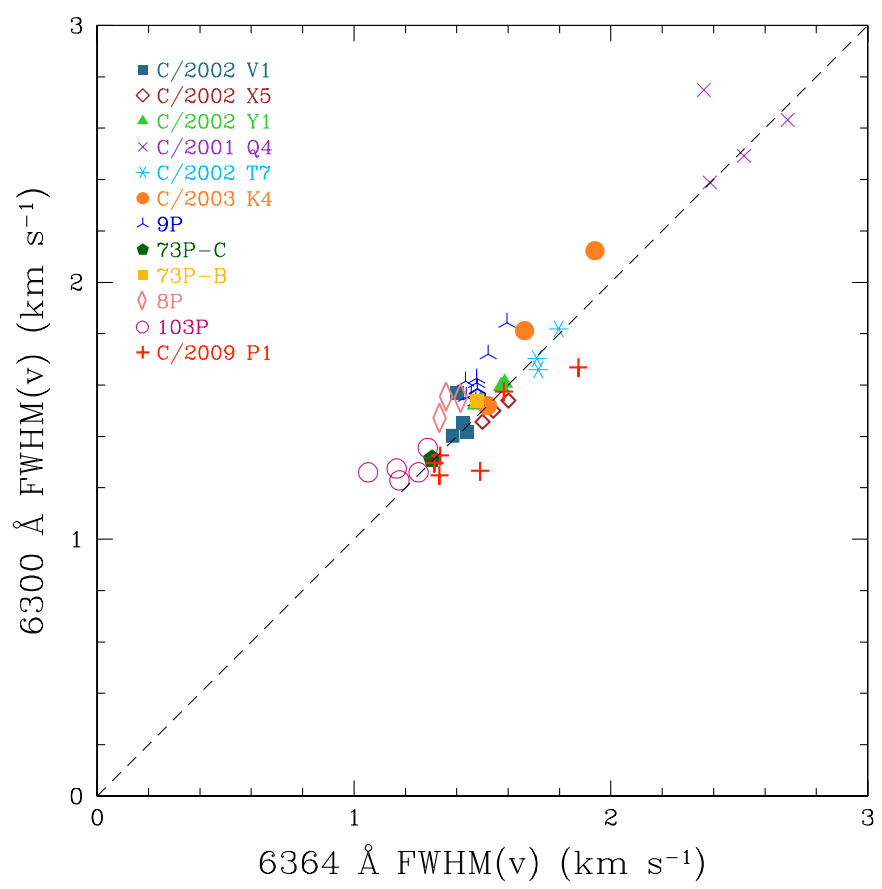

Fig. 8. The $6300.304 \AA \operatorname{FWHM}(v)$ against the $6363.776 \AA$ one. The different spectra for a given comet are denoted with the same symbol.

to those of other comets observed below 2.5 AU, and it gives a relative abundance of $\mathrm{CO}_{2}$ of only $\sim 3 \%$, while a $\mathrm{G} / \mathrm{R}$ ratio of $\sim 0.1$ to 0.2 would be needed to reach a $\mathrm{CO}_{2}$ abundance as high as $\sim 10 \%$ to $20 \%$.

We do not confirm a high value of $\mathrm{CO}_{2}$ abundance for 103P/Hartley 2 as claimed by McKay et al. (2013) or a large dispersion in the abundances. Their values might be higher because they used a $W_{\mathrm{H}_{2} \mathrm{O}}^{\text {green }}$ of Bhardwaj \& Raghuram (2012) equal to $2.6 \times 10^{-8} \mathrm{~s}^{-1}$, while the value provided in Table 1 of this paper is $6.4 \times 10^{-8} \mathrm{~s}^{-1}$.

The discrepancy with the EPOXI and ISO observations $\mathrm{CO}_{2}$ abundance is interesting. Below 2.5 AU, G/R values are distributed from 0.05 for comets like 9P/Tempel 1 and 8P/Tuttle

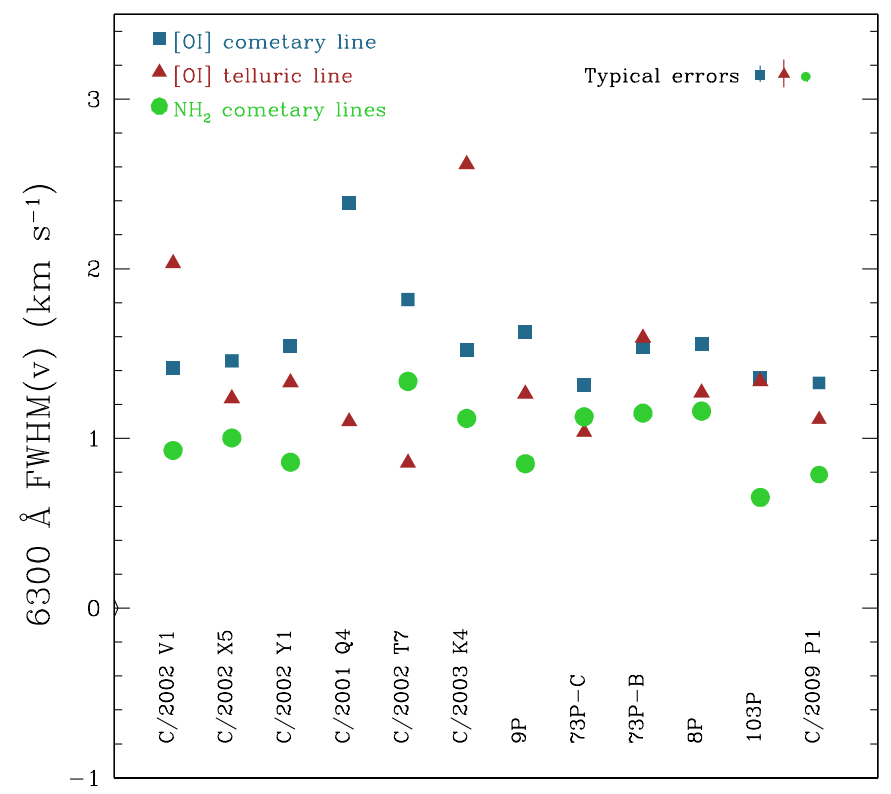

Fig. 9. The FWHM $(v)$ of the $6300.304 \AA[O I]$ cometary line (a), the [OI] telluric line $(\boldsymbol{\Lambda})$, and nearby $\mathrm{NH}_{2}$ cometary lines $(\bullet)\left(\mathrm{km} \mathrm{s}^{-1}\right)$ for one spectrum per comet. The typical errors correspond to the standard deviation $(\sigma)$ obtained considering all the spectra of comet $8 \mathrm{P} /$ Tuttle. We have also checked this error by measuring $\sigma$ randomly for a few spectra of other comets. For $\mathrm{C} / 2001 \mathrm{Q} 4$, the $\mathrm{NH}_{2}$ cometary lines are missing because no fluorescence lines were detected at such a large heliocentric distance.

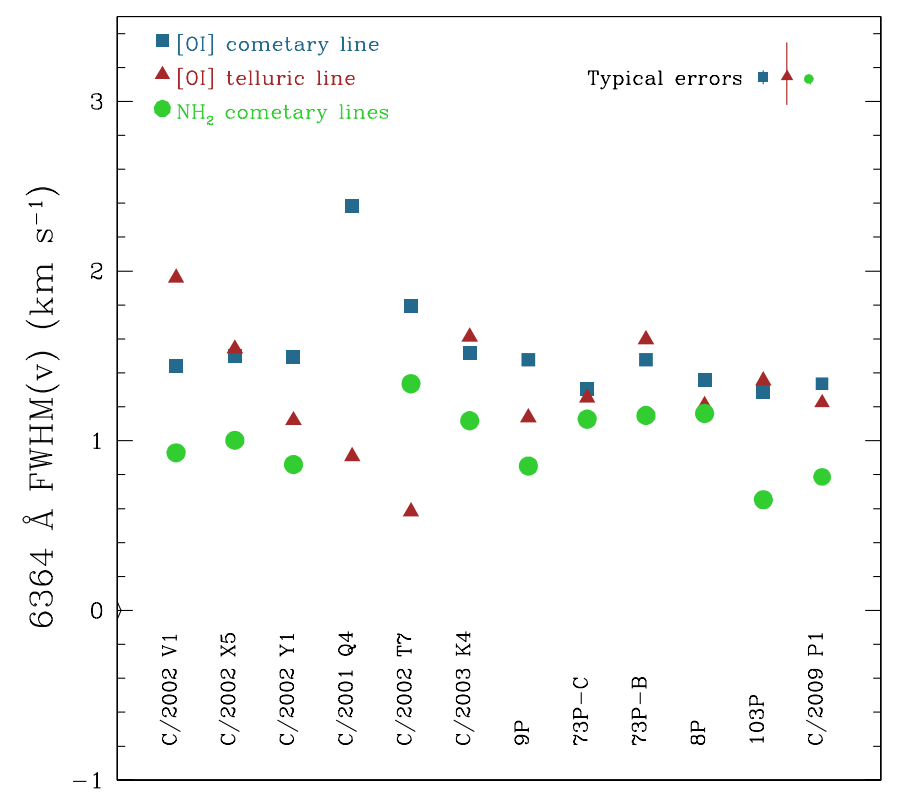

Fig. 10. Same as Fig. 9 for the $6363.776 \AA$ line.

and can go up to 0.12 for C/2002 T7 (LINEAR). The spread from comet to comet is higher than the errors estimated from the dispersion from the spectra of a same comet, which could be explained by an intrinsic variation in $G / R$ from comet to comet due to the content of $\mathrm{CO}_{2}$. The $\mathrm{G} / \mathrm{R}$ range, on the order of 0.1 (implying a $\mathrm{CO}_{2}$ variation of $\sim 20 \%$ ), is close to the range of $\mathrm{CO}_{2}$ abundance in comets of various origins measured below 2.5 AU (A'Hearn 2012). 
A. Decock et al.: [OI] lines in comets at various heliocentric distances

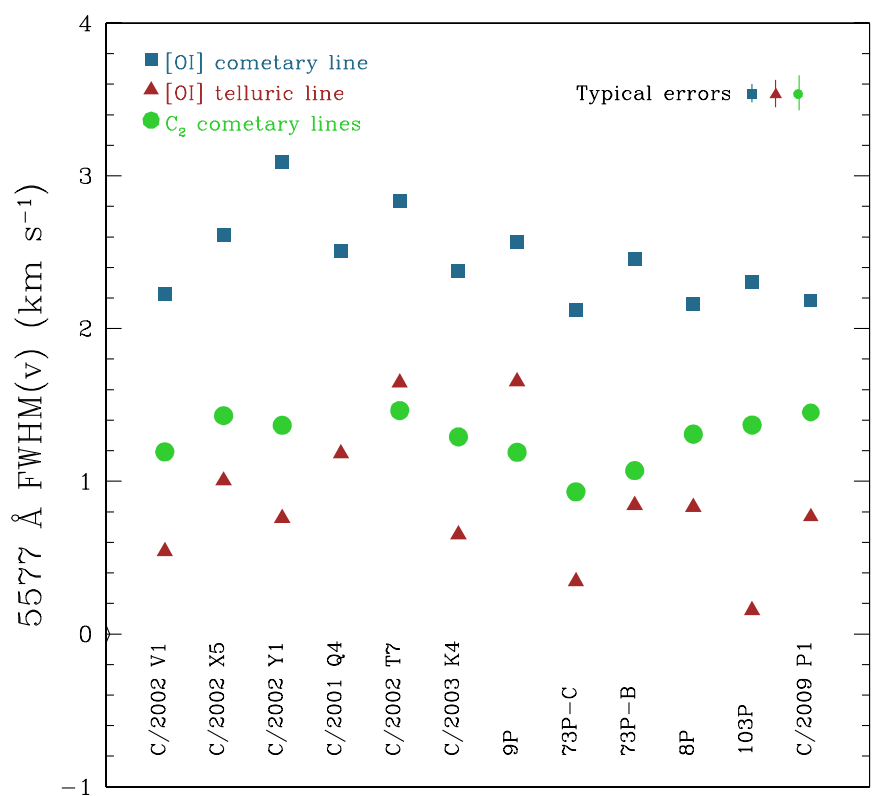

Fig. 11. Same as Fig. 9 for the $5577.339 \AA$ line and with $\mathrm{C}_{2}$ cometary lines.

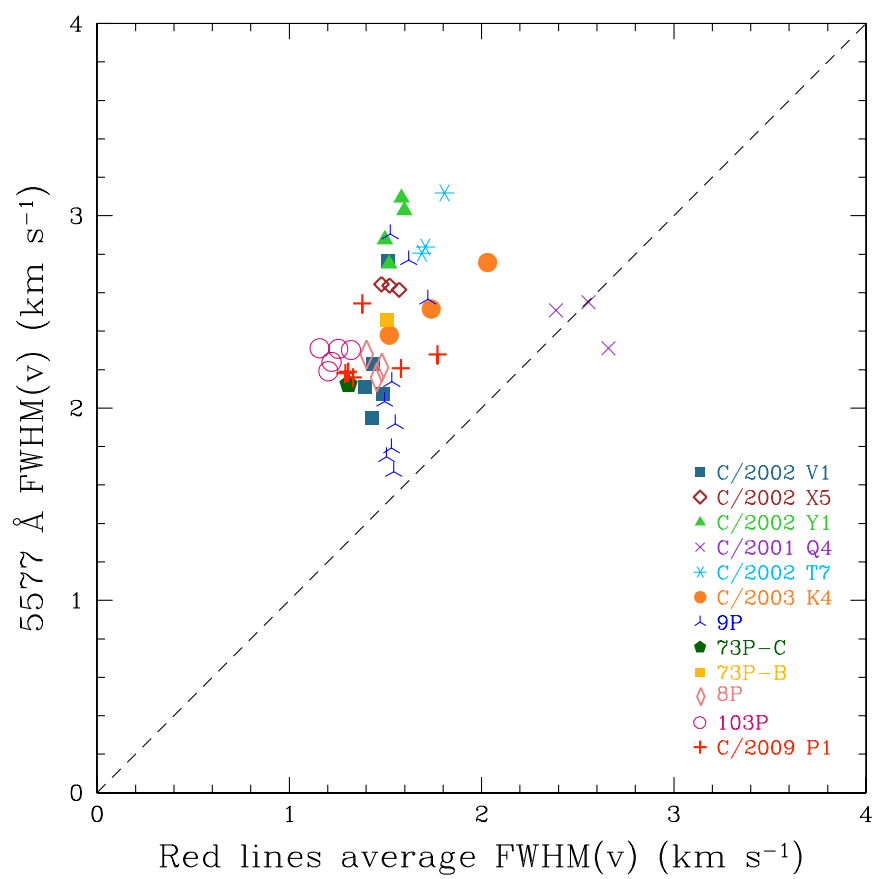

Fig. 12. The $5577.339 \AA$ line width against the average width of the red lines. The different spectra of a given comet are denoted by the same symbol. The green line is always larger than the red lines except for C/2001 Q4.

Some comets, such as 9P/Tempel 1 and $8 \mathrm{P} /$ Tuttle, have lower values than the pure water case (giving a $G / R_{\min }=0.08$ from Bhardwaj \& Raghuram (2012)) and that the 103P/Harley 2 $\mathrm{G} / \mathrm{R}$ ratio is too low compared to EPOXI $\mathrm{CO}_{2}$ abundances could indicate a problem with the models. The pure water ratio should be 0.05 or lower based on the comets with the lowest values. 9P/Tempel 1 has a $\mathrm{CO}_{2}$ abundance of $\sim 7 \%$ (Feaga et al. (2007a), Feaga et al. (2007b). Bhardwaj \& Raghuram (2012) tried $\alpha_{\mathrm{H}_{2} \mathrm{O}}^{\text {green }}$ values from $0 \%$ to $1 \%$ and finally chose $1 \%$, but this value is uncertain. Without $\mathrm{CO}_{2}, \mathrm{G} / \mathrm{R}_{\min }=\beta^{\text {green }} W_{\mathrm{H}_{2} \mathrm{O}}^{\text {green }} / W_{\mathrm{H}_{2} \mathrm{O}}^{\text {red }}$.

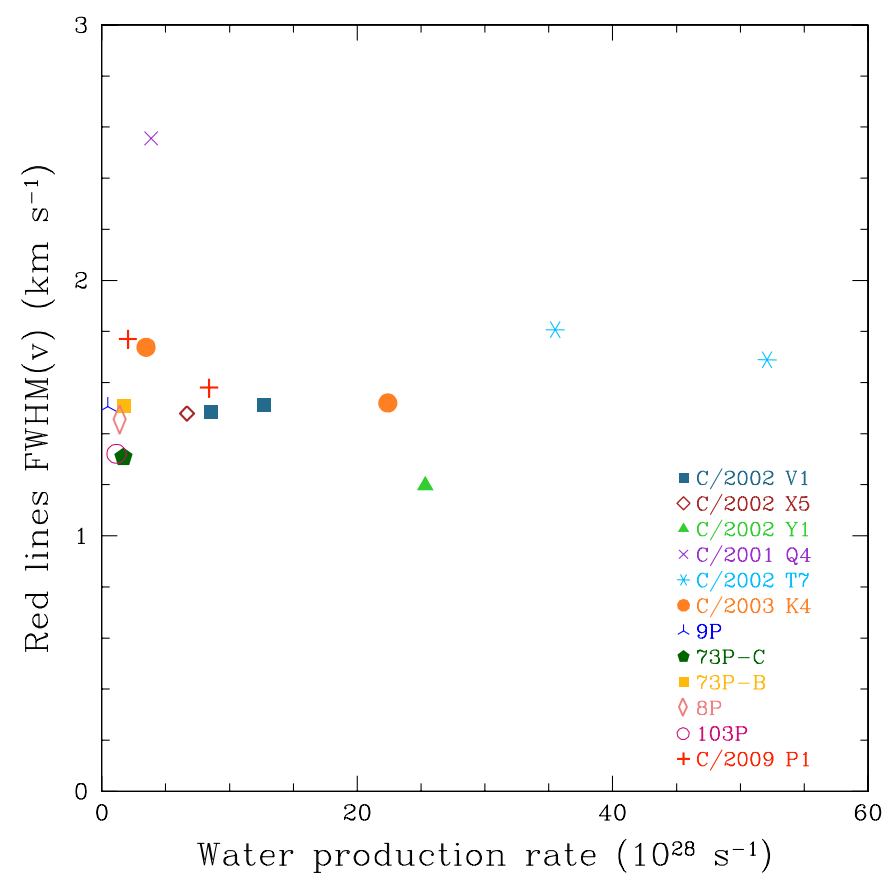

Fig. 13. Average of the $[\mathrm{OI}]$ red lines FWHM versus the water production rate.

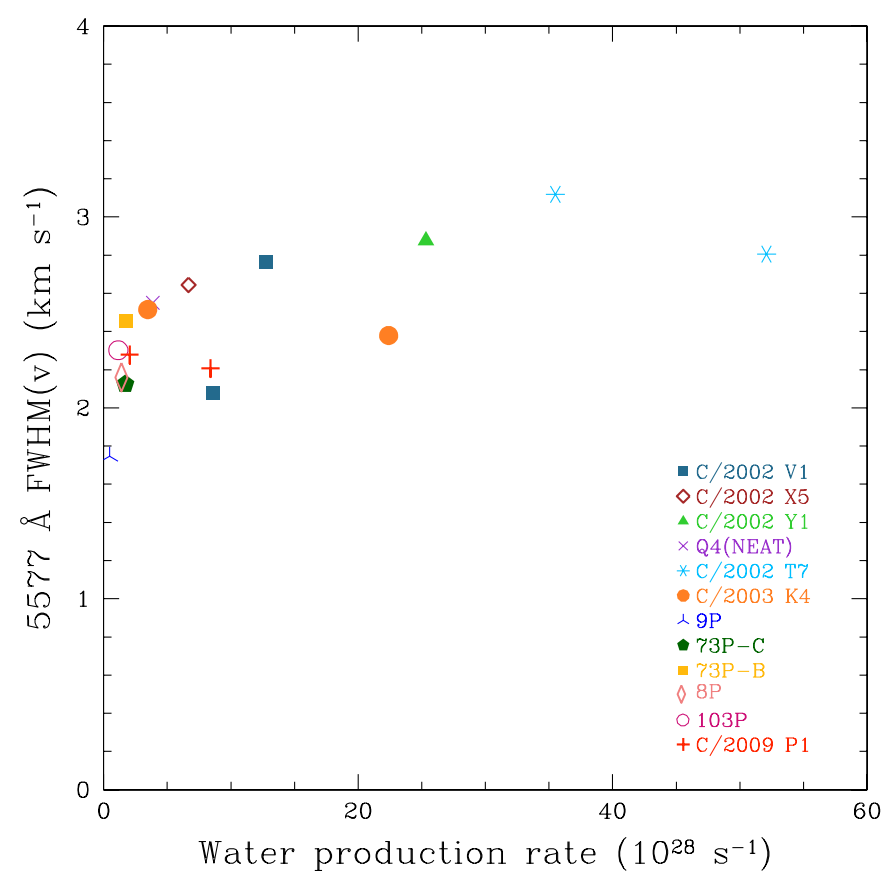

Fig. 14. Same as Fig. 13 but for the $5577.339 \AA$ Aine.

If we use $0.5 \%$ for $\alpha_{\mathrm{H}_{2} \mathrm{O}}^{\text {green }}, \mathrm{G} / \mathrm{R}_{\min }$ is equal to 0.04 instead of 0.08 , which would be in better agreement with our lowest values.

\subsection{Deep impact}

The spectra of comet 9P/Tempel 1 were analyzed before and after the July 4 collision with the Deep Impact spacecraft. No difference was observed in the intensity ratios and the lines widths before and after the impact (Fig. 20). We have no spectrum during the impact. The first spectrum after the impact was taken at 


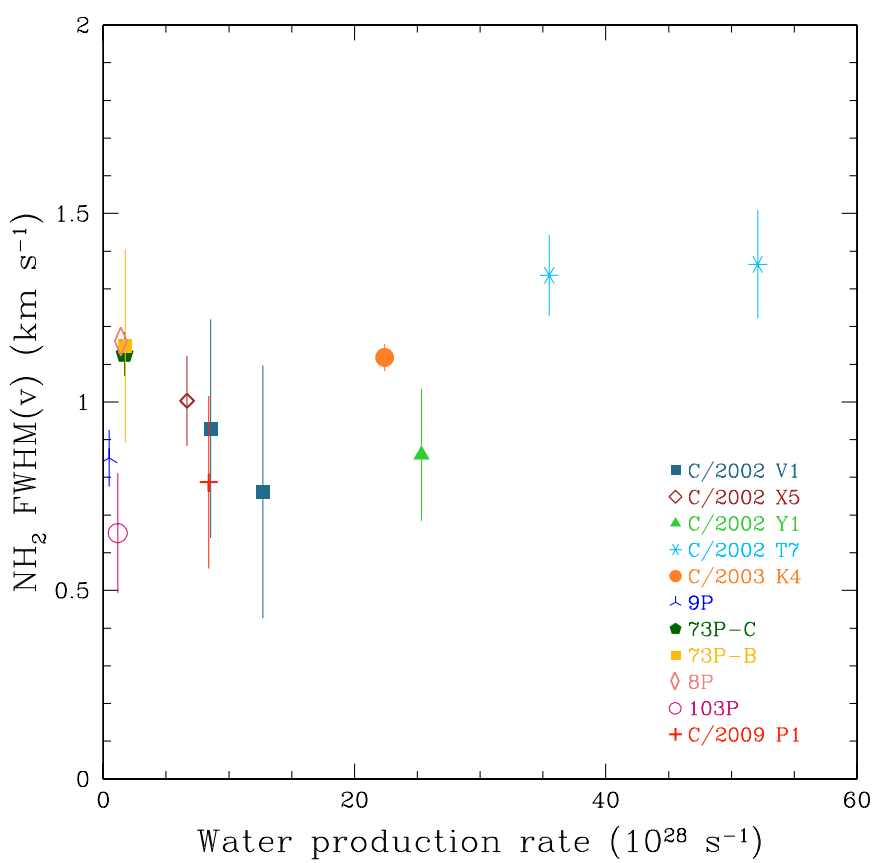

Fig. 15. Same as Fig. 13 but for the $\mathrm{NH}_{2}$ line.

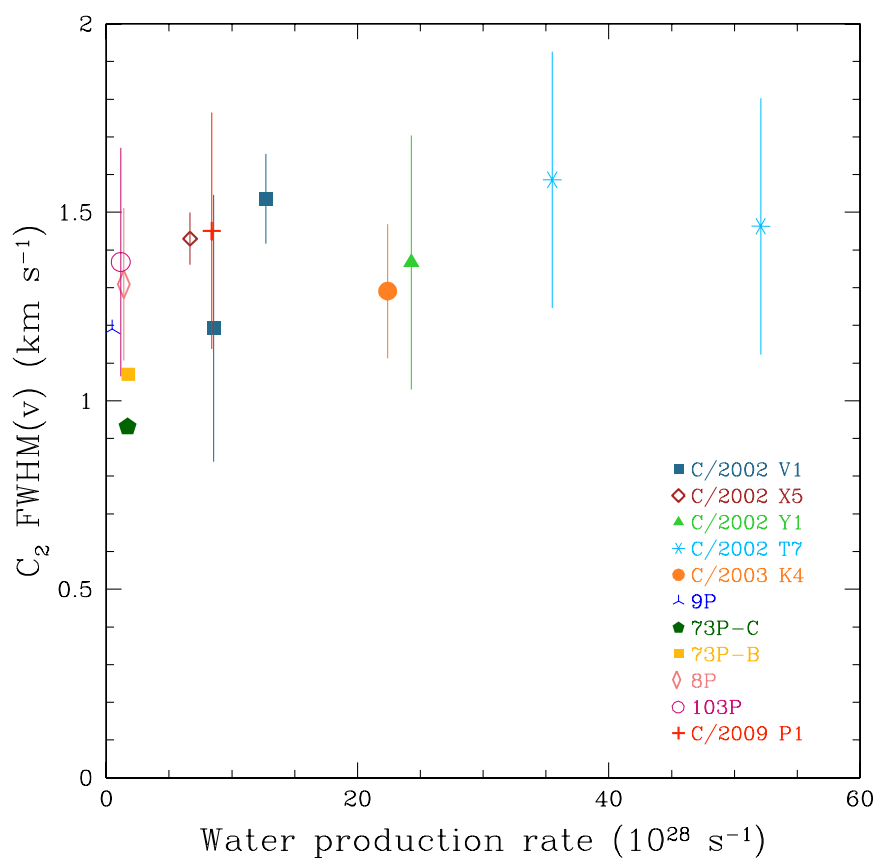

Fig. 16. Same as Fig. 13 but for the $C_{2}$ line.

2453559.954 UT (i.e. $\sim 6 \mathrm{~h}$ after the impact). Cochran (2008) did not notice any change during the impact either.

\subsection{Solar activity}

Our data were obtained over the $23^{\mathrm{d}}$ solar cycle characterized by its maximum of activity in 2001 and its minimum activity in 2008. Despite the decrease in the solar activity during the observations of C/2001 V1 (NEAT) and 8P/Tuttle, no change in the intensities and widths of lines were noticed. The solar activity does not appear to have any influence on the results of our work.
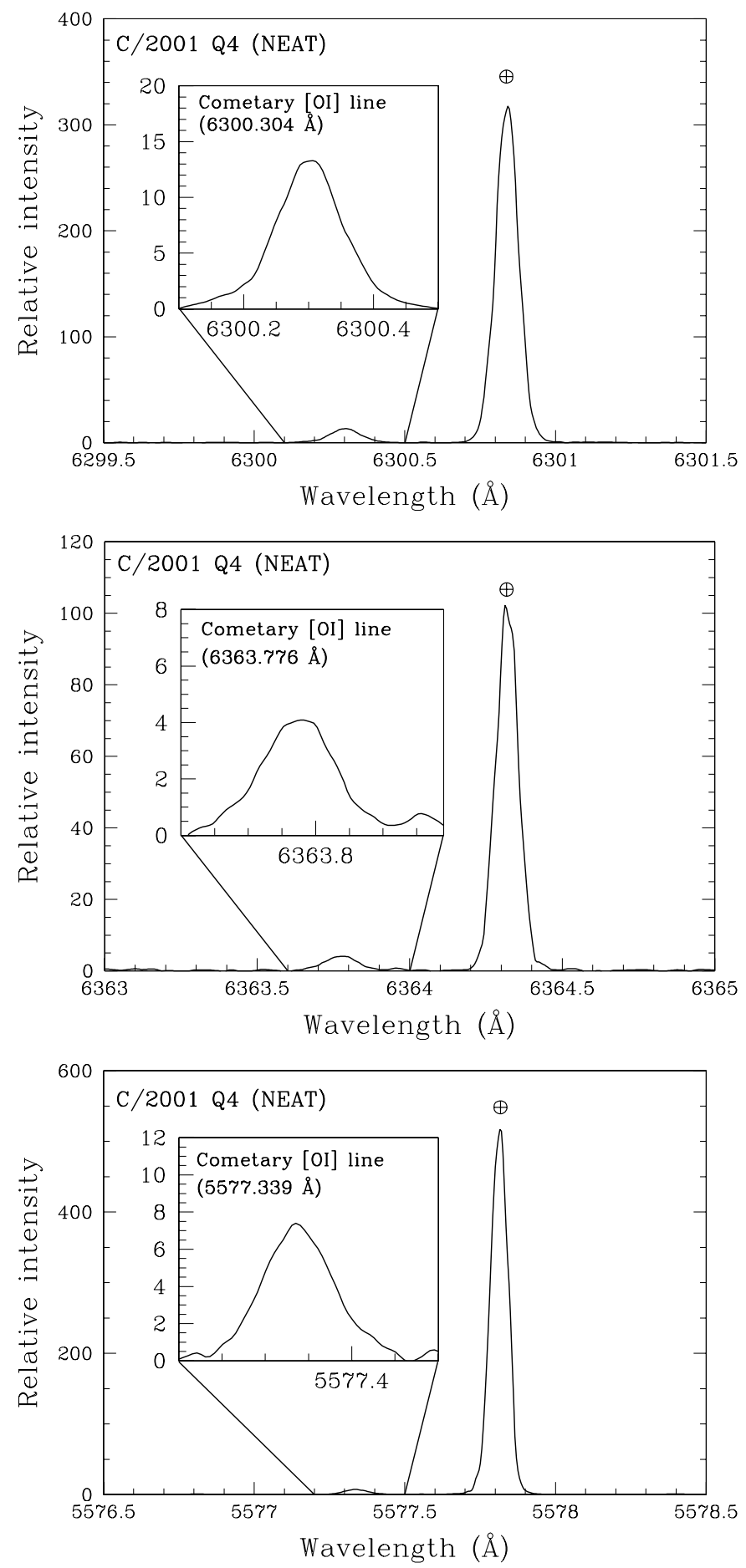

Fig. 17. Two hours spectra of comet C/2001 Q4 obtained with the ESO VLT. These spectra are the most distant detection of [OI] lines in a comet (at 3.7 AU). The lines are well detected and well separated from the telluric line. The width of the green and the red lines are the same.

\section{Conclusion}

From January 2003 to September 2011, 12 comets of various origins were observed at different heliocentric distances, and 48 high-resolution spectra were obtained with the UVES spectrograph at VLT (ESO). Within this whole sample, we observed the three $[\mathrm{OI}]$ forbidden oxygen lines with high resolution and signal-to-noise and we measured the intensity ratios $\left(I_{6300} / I_{6364}\right.$ 
A. Decock et al.: [OI] lines in comets at various heliocentric distances

Table 8. Water production rates and their references.

\begin{tabular}{lccccc}
\hline \hline Comet & $r$ & Spectra observed date & $Q_{\mathrm{H}_{2} \mathrm{O}}\left(10^{28} \mathrm{~s}^{-1}\right)$ & Date & Reference for $Q_{\mathrm{H}_{2} \mathrm{O}}$ \\
\hline C/2002 V1 (NEAT) & 1.22 & 2003 Jan. 8 & 8.56 & 2003 Jan. 8 & Combi et al. (2011) \\
& 1.01 & 2003 Mar. 21 & 12.73 & 2003 Mar. 21 & Combi et al. (2011) \\
C/2002 X5 (Kudo-Fujikawa) & 1.06 & 2003 Feb. 19 & 6.67 & 2003 Feb. 19 & Combi et al. (2011) \\
C/2002 T7 (LINEAR) & 0.68 & 2004 May 6 & 35.5 & 2004 May 5 & DiSanti et al. (2006) \\
& 0.94 & 2004 May 27 & 52.1 & 2004 May 27 & Combi et al. (2009) \\
C/2003 K4 (LINEAR) & 2.61 & 2004 May 6 & 3.47 & 2004 May 6 & 1 \\
& 1.20 & 2004 Nov. 20 & 22.4 & 2004 Nov. 20 & Gicquel et al. (2012) \\
9P/Tempel 1 & 1.51 & 2005 Jul. 3 & 0.47 & 2005 Jul. 3 & Gehleicher \& Bair (2011) \\
73P-C/Schwassmann-Wachmann 3 & 095 & 2006 May 27 & 1.70 & 2006 May 17 & Scher 2011$)$ \\
73P-B/Schwassmann-Wachmann 3 3 & 0.94 & 2006 Jun. 12 & 1.76 & 2006 May 18 & Schleicher \& Bair (2011) \\
8P/Tuttle & 1.04 & 2008 Jan. 16 & 1.4 & 2008 Jan. 3 & Barber et al. (2009) \\
103P/Hartley 2 & 1.06 & 2010 Nov. 5 & 1.15 & 2010 Nov. 31 & Knight \& Schleicher (2013) \\
C/2009 P1 (Garradd) & 2.07 & 2011 Sep. 12 & 8.4 & 2011 Sep. 17-21 & Paganini et al. (2012) \\
\hline
\end{tabular}

Notes. We could find $Q_{\mathrm{H}_{2} \mathrm{O}}$ data measured very close to our observing dates. ${ }^{1}$ These water production rates were evaluated using the Jorda relation (Jorda et al. 1991) with the magnitudes given by Manfroid et al. (2005).

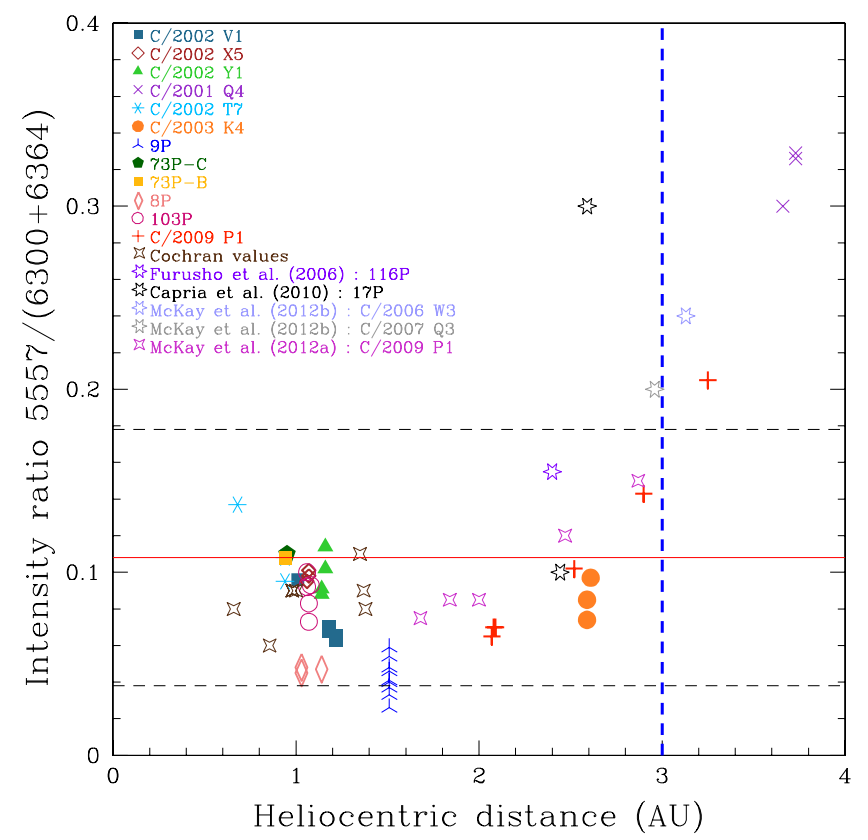

Fig. 18. The $G / R$ intensity ratio as a function of the heliocentric distance (AU). The same symbol is used for the different spectra of each comet. The most distant comets have higher ratios. Other values from the literature are also plotted. The solid line represents the average value only for our sample of spectra, and the standard deviation $(\sigma)$ range is denoted with horizontal dashed lines. If we only consider the data at $r<2 \mathrm{AU}$, the average value of the $\mathrm{G} / \mathrm{R}$ ratio is $0.09 \pm 0.02$. The vertical dashed line at $3 \mathrm{AU}$ represents the distance out to which the sublimation of water is strongly decreasing (Crovisier \& Encrenaz 2000).

and $\left.\mathrm{G} / \mathrm{R}=I_{5577} /\left(I_{6300}+I_{6364}\right)\right)$, as well as the FWHM $(v)$ of the lines. The results can be summarized as follows.

1. The $I_{6300} / I_{6364}$ ratio $(3.11 \pm 0.10)$ is in very good agreement with the branching ratio obtained by quantum mechanics, especially the work done by Galavis et al. (1997). However, some systematic errors on the order of 0.1 are possible so that we cannot exclude the new value of Storey \& Zeippen (2000).

2. From theoretical values given in Table 1 , the $\mathrm{G} / \mathrm{R}$ ratio for comets observed below $2 \mathrm{AU}(0.09 \pm 0.02)$ confirms $\mathrm{H}_{2} \mathrm{O}$ as the main parent molecule photodissociating to produce

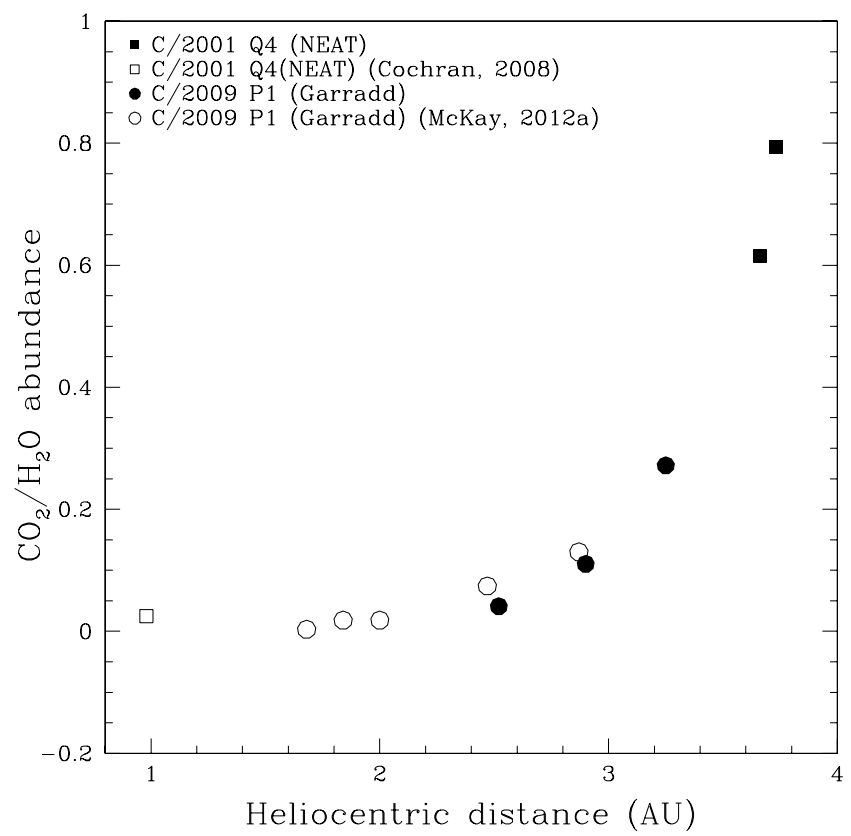

Fig. 19. Evolution of the $\mathrm{CO}_{2} / \mathrm{H}_{2} \mathrm{O}$ abundance as a function of the heliocentric distance $(\mathrm{AU})$. Assuming that a higher $\mathrm{G} / \mathrm{R}$ ratio comes from a more important contribution of $\mathrm{CO}_{2}$ for large heliocentric distance comets, and measuring this ratio can give us the $\mathrm{CO}_{2}$ abundance from ground observations.

Table 9. The $\mathrm{CO}_{2} / \mathrm{H}_{2} \mathrm{O}$ abundances estimated from Eq. (12) for the comet 103P/Hartley 2.

\begin{tabular}{lccr}
\hline \hline $\mathrm{JD}-2450000.5$ & $r(\mathrm{AU})$ & $\mathrm{G} / \mathrm{R}$ & $N_{\mathrm{CO}_{2}} / N_{\mathrm{H}_{2} \mathrm{O}}$ \\
\hline 5505.288 & 1.06 & 0.09 & 0.028 \\
5505.328 & 1.06 & 0.10 & 0.040 \\
5510.287 & 1.07 & 0.07 & 0.001 \\
5510.328 & 1.07 & 0.08 & 0.015 \\
5511.363 & 1.08 & 0.09 & 0.030 \\
\hline
\end{tabular}

oxygen atoms. However, when the comet is located at greater heliocentric distances $(r \geq 2.5 \mathrm{AU})$, the ratio increases rapidly, showing that another parent molecule is contributing. We have shown that $\mathrm{CO}_{2}$ is the best candidate. 


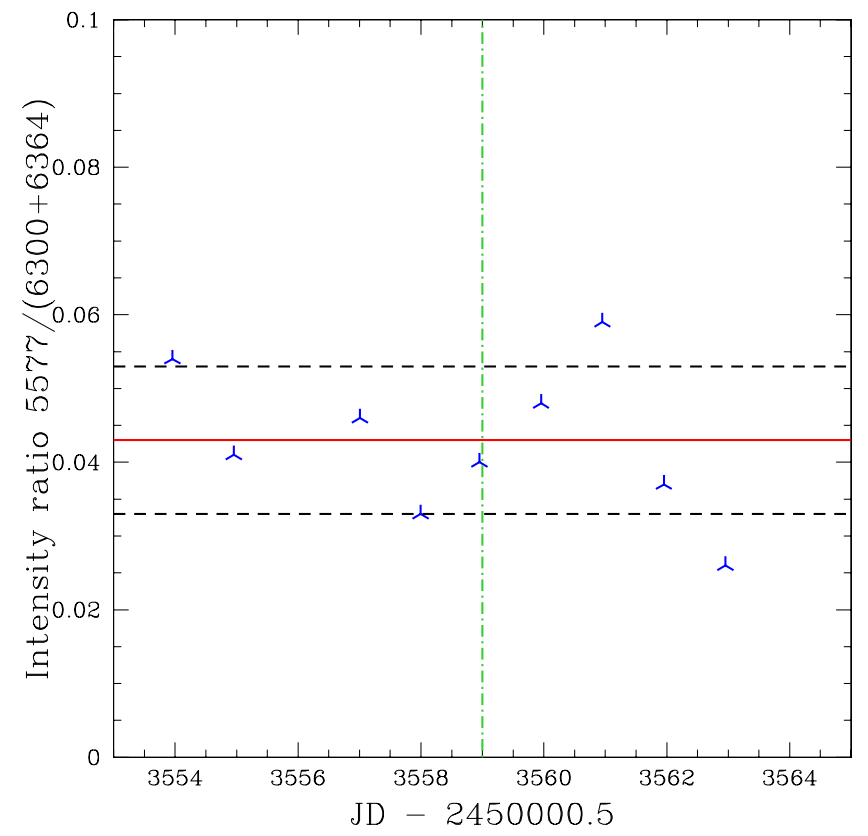

Fig. 20. The $G / R$ intensity ratio of comet $9 P / T e m p e l ~ 1$ from JD 2453553.955 to 2453562.956 UT. The Deep Impact event corresponds to the vertical line (53559 UT). The average value is shown by the solid red line and the dashed lines correspond to the standard deviation $(\sigma)$. The mean value around the impact (from 2453553.955 UT to $2453562.956 \mathrm{UT}$ ) is $0.04 \pm 0.01$. No variation of the ratio was noticed after the impact.

Measuring the $\mathrm{G} / \mathrm{R}$ ratios could then be a new way to estimate the abundances of $\mathrm{CO}_{2}$, a very difficult task from the ground. Assuming that only the photo-dissociation of $\mathrm{H}_{2} \mathrm{O}$ and $\mathrm{CO}_{2}$ produce [OI], we found a relation between $\mathrm{CO}_{2}$ abundance and the heliocentric distance of the comet. The $\mathrm{C} / 2001 \mathrm{Q} 4$ (NEAT) abundance of $\mathrm{CO}_{2}$ at $3.7 \mathrm{AU}$ is found to be $\sim 75 \%$.

3. The intrinsic green line width is wider than the red ones by about $1 \mathrm{~km} \mathrm{~s}^{-1}$. Theoretical estimations considering Ly- $\alpha$ as the only excitation source for the two states led to the conclusion that the excess energy for $\mathrm{O}\left({ }^{1} \mathrm{D}\right)$ is greater than for $\mathrm{O}\left({ }^{1} \mathrm{~S}\right)$, which contradicts our observations. This discrepancy might be explained by the different nature of the excitation source and/or a contribution of $\mathrm{CO}_{2}$ as parent molecule to the $\mathrm{O}\left({ }^{1} \mathrm{~S}\right)$ state. Indeed, Bhardwaj \& Raghuram (2012) have shown that for the photodissociation of $\mathrm{CO}_{2}$, the main excitation source might instead be the 995-1165 $\AA$ band. To check this hypothesis quantitatively, it would be necessary to theoretically estimate the excess energy for the oxygen atoms when the wavelength band is 995-1165 $\AA$, accounting for the photodissociation of both $\mathrm{H}_{2} \mathrm{O}$ and $\mathrm{CO}_{2}$. The widths of the three [OI] lines are similar in $\mathrm{C} / 2001 \mathrm{Q} 4$ at $~ 3.7 \mathrm{AU}$. This could agree with $\mathrm{CO}_{2}$ being the main contributor for the three $[\mathrm{OI}]$ lines at a large heliocentric distance. Other comets at large $r$ have to be observed to test this hypothesis.

4. More $\mathrm{CO}_{2}$ (and $\mathrm{CO}$ ) abundance determinations, together with $\mathrm{G} / \mathrm{R}$ oxygen ratios and line widths at different heliocentric distances, are clearly needed in order to give a general conclusion about the oxygen parent molecule.

5. The $\mathrm{CO}_{2}$ rich comet $103 \mathrm{P} /$ Hartley $2(\sim 20 \%)$ does not present a high $\mathrm{G} / \mathrm{R}$ ratio normally expected from Eq. (12). We suggest a new value of $0.5 \%$ for $\alpha_{\mathrm{H}_{2} \mathrm{O}}^{\text {green }}$ that could also explain the low values of $\mathrm{G} / \mathrm{R}$ obtained for comets $9 \mathrm{P} /$ Tempel 1 and $8 \mathrm{P} /$ Tuttle.

Acknowledgements. A.D. acknowledges the support of the Belgian National Science Foundation F.R.I.A., Fonds pour la formation à la Recherche dans l'Industrie et l'Agriculture. E.J. is Research Associate FNRS, D.H. is Senior Research Associate at the FNRS and J.M. is Research Director of the FNRS. C. Arpigny is acknowledged for the helpful discussions and constructive comments.

\section{References}

A'Hearn, M. F. 2012, in AAS Meeting Abstracts, 220, 120.04

A'Hearn, M. F., Belton, M. J. S., Delamere, W. A., et al. 2011, Science, 332, 1396

Barber, R. J., Miller, S., Dello Russo, N., et al. 2009, MNRAS, 398, 1593

Bhardwaj, A., \& Raghuram, S. 2012, ApJ, 748, 13

Biver, N., Bockelée-Morvan, D., Crovisier, J., et al. 1999, AJ, 118, 1850

Biver, N., Bockelée-Morvan, D., Lis, D. C., et al. 2012, LPI Contributions, 1667, 6330

Capria, M. T., Cremonese, G., \& de Sanctis, M. C. 2010, A\&A, 522, A82

Cochran, A. L. 2008, Icarus, 198, 181

Cochran, A. L., \& Cochran, W. D. 2001, Icarus, 154, 381

Combi, M. R., Mäkinen, J. T. T., Bertaux, J.-L., Lee, Y., \& Quémerais, E. 2009, AJ, 137, 4734

Combi, M. R., Boyd, Z., Lee, Y., et al. 2011, Icarus, 216, 449

Crovisier, J., \& Encrenaz, T. 2000, Comet science: the study of remnants from the birth of the solar system (CUP)

Crovisier, J., Encrenaz, T., Lellouch, E., et al. 1999, in The Universe as Seen by ISO, eds. P. Cox, \& M. Kessler, ESA Spec. Publ., 427, 161

Decock, A., Jehin, E., Manfroid, J., \& Hutsemékers, D. 2011, in EPSC-DPS Joint Meeting, 2011, 1126

Dekker, H., D’Odorico, S., Kaufer, A., Delabre, B., \& Kotzlowski, H. 2000, in SPIE Conf. Ser. 4008, eds. M. Iye, \& A. F. Moorwood, 534

DiSanti, M. A., Bonev, B. P., Magee-Sauer, K., et al. 2006, ApJ, 650, 470

Ehrenfreund, P., \& Charnley, S. B. 2000, ARA\&A, 38, 427

Feaga, L. M., A’Hearn, M. F., Sunshine, J. M., Groussin, O., \& Farnham, T. L. 2007a, Icarus, 190, 345

Feaga, L. M., A’Hearn, M. F., Sunshine, J. M., Groussin, O., \& Farnham, T. L. 2007b, Icarus, 191, 134

Feldman, P. D., Weaver, H. A., Christian, D., et al. 2004, in BAAS, AAS/Division for Planetary Sciences Meeting Abstracts 36, 1121

Festou, M. C. 1981, A\&A, 96, 52

Festou, M., \& Feldman, P. D. 1981, A\&A, 103, 154

Furusho, R., Kawakita, H., Fuse, T., \& Watanabe, J. 2006, Adv. Space Res., 38, 1983

Galavis, M. E., Mendoza, C., \& Zeippen, C. J. 1997, A\&AS, 123, 159

Gicquel, A., Bockelée-Morvan, D., Zakharov, V. V., et al. 2012, A\&A, 542, A119 Huebner, W. F., \& Carpenter, C. W. 1979, NASA STI/Recon Technical Report N, 80, 24243

Jehin, E., Manfroid, J., Hutsemékers, D., Arpigny, C., \& Zucconi, J.-M. 2009, Earth Moon Planets, 105, 167

Jorda, L., Crovisier, J., \& Green, D. W. E. 1991, LPI Contributions, 765, 108

Knight, M. M., \& Schleicher, D. G. 2013, Icarus, 222, 691

Levison, H. F. 1996, in Completing the Inventory of the Solar System, eds. T. Rettig, \& J. M. Hahn, ASP Conf. Ser., 107, 173

Manfroid, J., Jehin, E., Hutsemékers, D., et al. 2005, A\&A, 432, L5

Manfroid, J., Jehin, E., Hutsemékers, D., et al. 2009, A\&A, 503, 613

McKay, A. J., Chanover, N. J., DiSanti, M. A., et al. 2012a, LPI Contributions, 1667,6212

McKay, A. J., Chanover, N. J., Morgenthaler, J. P., et al. 2012b, Icarus, 220, 277

McKay, A. J., Chanover, N. J., Morgenthaler, J. P., et al. 2013, Icarus, 222, 684

Morrison, N. D., Knauth, D. C., Mulliss, C. L., \& Lee, W. 1997, PASP, 109, 676

Ootsubo, T., Kawakita, H., Hamada, S., et al. 2012, ApJ, 752, 15

Paganini, L., Mumma, M. J., Villanueva, G. L., et al. 2012, ApJ, 748, L13

Schleicher, D. G., \& Bair, A. N. 2011, AJ, 141, 177

Slanger, T. G., Cosby, P. C., Sharpee, B. D., Minschwaner, K. R., \& Siskind, D. E. 2006, J. Geophys. Res. (Space Physics), 111, 12318

Storey, P. J., \& Zeippen, C. J. 2000, MNRAS, 312, 813

Swings, P. 1962, Ann. Astrophys., 25, 165

Tseng, W.-L., Bockelée-Morvan, D., Crovisier, J., Colom, P., \& Ip, W.-H. 2007, A\&A, 467, 729

Weaver, H. A., Feldman, P. D., A'Hearn, M. F., Dello Russo, N., \& Stern, S. A. 2011, ApJ, 734, L5 